\title{
Transport by gravito-inertial waves in differentially rotating stellar radiation zones
}

\section{I - Theoretical formulation}

\author{
S. Mathis ${ }^{1,2}$ \\ ${ }^{1}$ Laboratoire AIM, CEA/DSM-CNRS-Université Paris Diderot, IRFU/SAp Centre de Saclay, 91191 Gif-sur-Yvette, France \\ e-mail: stephane.mathis@cea.fr \\ ${ }^{2}$ LUTH, Observatoire de Paris-CNRS-Université Paris Diderot, Place Jules Janssen, 92195 Meudon, France \\ Received 8 July 2008 / Accepted 3 July 2009
}

ABSTRACT

\begin{abstract}
Context. We examine the dynamics of low-frequency waves in differentially rotating stellar radiation zones, the angular velocity being taken as generally as possible depending both on radius and on latitude in stellar interiors. The associated induced transport of angular momentum, which plays a key role in the evolution of rotating stars, is derived.

Aims. We focus on the wave-induced transport of angular momentum, taking into account the Coriolis acceleration in the case of strong radial and latitudinal differential rotation. We thus go beyond the "weak differential rotation" approximation, where rotation is almost a solid-body one plus a residual radial differential rotation. As has been shown in previous works, the Coriolis acceleration modifies such transport.

Methods. We built analytically a complete formalism that allows the study of rotational transport in stellar radiation zones taking into account the wave action modified by a general strong differential rotation.

Results. The different approximations possible for low-frequency waves in a differentially rotating stably stratified radiative region, namely the traditional and the JWKB approximations, are examined and discussed. The complete bidimensional structure of regular elliptic gravito-inertial waves, which verify these approximations, is derived and compared to those in the "weak differential rotation" case. Next, associated transport of energy and of angular momentum in the vertical and in the horizontal directions and the dynamical equations, respectively for the mean radial differential rotation and the latitudinal one, are obtained.

Conclusions. The complete formalism, which takes into account low-frequency wave action, is derived and can be used for the study of secular hydrodynamics of radiative regions and of the associated mixing. The modification of waves due to general strong differential rotation and their feed-back on the angular momentum transport are treated rigourously. In a forthcoming paper (Paper II), this formalism will be applied to the case of solar differential rotation. However, the case of hyperbolic gravito-inertial waves should be carefully studied.
\end{abstract}

Key words. hydrodynamics - waves - turbulence - methods: analytical - stars: rotation - stars: evolution

\section{Introduction}

The study of helioseismology, asteroseismology and powerful ground-based instrumentation dedicated to stellar physics is developing strongly (Turck-Chièze 2005, 2006, 2008; Aerts et al. 2008, and references therein) generating tight constraints on the internal structure and dynamics of stars. This is the reason why it is now necessary to build stellar models that take into account the dynamical processes from the birth of stars to their death.

A coherent picture of the dynamics of stellar radiation zones where the non-standard chemicals mixing takes place is thus required (cf. Zahn 2005).

A complex transport, which involves several mechanisms, takes place in these regions. First, rotation induces a large-scale circulation, the called meridional circulation, which acts to simultaneously transport angular momentum, chemicals and the magnetic field by advection. This circulation is due to differential rotation, to structural adjustments and to angular momentum losses at the surface (cf. Busse 1982; Zahn 1992; Talon et al. 1997; Maeder \& Zahn 1998; Meynet \& Maeder 2000; Garaud 2002b; Palacios et al. 2003-2006; Mathis \& Zahn 2004, 2005; Rieutord 2006; Espinosa Lara \& Rieutord 2007; Mathis et al. 2007; Decressin et al. 2009).
Next, differential rotation induces hydrodynamical turbulence through various instabilities such as the shear, the baroclinic and the multidiffusive ones. As in the terrestrial atmosphere, this turbulence acts to reduce its cause, namely the gradients of angular velocity (cf. Zahn 1983; Talon \& Zahn 1997; Garaud 2001; Maeder 2003; Mathis et al. 2004).

On the other hand, rotation interacts with fossil magnetic fields. Then, the mean secular torque of the Lorentz force and the magnetohydrodynamical instabilities such as the Tayler-Spruit and the multidiffusive magnetic instabilities modify the transport of angular momentum and of chemicals (cf. Charbonneau \& Mac Gregor 1993; Gough \& McIntyre 1998; Garaud 2002a; Spruit 1999-2002; Menou et al. 2004; Maeder \& Meynet 2004; Eggenberger et al. 2005; Braithwaite \& Spruit 2005; Braithwaite 2006; Brun \& Zahn 2006; Zahn et al. 2007).

Finally, internal gravity waves (hereafter IGWs), which are excited at the borders with convective zones, propagate through radiative regions where they extract or deposit angular momentum at the location where they are damped, leading to a modification of the angular velocity profile and consequently of the chemical distribution (cf. Goldreich \& Nicholson 1989; Schatzman 1993; Kumar \& Quataert 1997; Zahn et al. 1997; Ringot 1998; Talon et al. 2002; Talon \& Charbonnel 2005; Rogers \& Glatzmaier 2005b, 2006). 
Here, we focus on IGWs. It is likely that there is a magnetic field in stellar radiation zones and more particularly at the level of the tachocline(s), at the border(s) between convection and radiation, which may be the zone of the storage of the mean axisymmetric part of the field toroidal component. In the presence of such a magnetic field, IGWs are then magneto-gravito-inertial waves (which are often called Magnetic Archimedean Coriolis (MAC) waves in geophysics) and the field acts as a filter to their vertical propagation (cf. Schatzman 1993; Barnes et al. 1998). In this work, we choose as a first step to ignore this interaction between IGWs and the magnetic field, which will be studied in a forthcoming paper, and to focus on purely hydrodynamical gravito-inertial waves.

In this context, it has now been undertaken to go beyond the non-rotating approximation in the treatment of IGWs propagation and induced transport. The Coriolis acceleration which strongly modifies IGWs as soon as $\sigma \sim 2 \Omega$ (where $\sigma$ and $2 \Omega$ are respectively the wave's frequency and the inertial one) is then taken into account. Depending on the excited wave spectrum which is assumed (cf. Kumar et al. 1999; Rogers \& Glatzmaier 2005b, 2006; Rogers et al. 2008, and the detailed discussion in Sect. 4.2.1.), the Coriolis acceleration effects have thus to be studied in a non-perturbative way (cf. Fig. 2) mainly for lowfrequency gravito-inertial waves which may be excited in stellar radiation zones in the neighborhood of the inertial frequency $(2 \Omega)$.

To achieve this aim, the Coriolis acceleration has first been treated using the traditional approximation, that can be applied in stellar radiation zones in the super-inertial regime where $2 \Omega<$ $\sigma \ll N$ in the case of uniform rotation ( $N$ is the Brunt-Väisälä frequency) (see for example Berthomieu et al. 1978; Friedlander 1987; Talon 1997; Mathis 2005). First numerical results using a stellar evolution code have been obtained (cf. Pantillon et al. 2007; Mathis et al. 2008).

However, in those previous works, a strong approximation on the differential rotation profile is assumed. In fact, the approximation of a weak differential rotation, where the rotation must be almost a solid-body one plus a residual radial differential rotation, is chosen in order to use the formalism coming from the treatment of Earth and planetary tides (Eckart 1960, Miles 1974). This is an imperative first step to understand the way in which the Coriolis acceleration modifies the transport due to IGWs.

Nevertheless, this approximation has to be relaxed in the case of a real star where strong gradients of angular velocity can appear, both in the radial and in the latitudinal directions, due to angular momentum transport. First, as shown by Talon \& Charbonnel (2005), strong radial $\Omega$-gradients are created during the wave-induced angular momentum extraction. Moreover, the angular velocity of the regions of waves excitation at the borders of radiative regions with adjacent convection zones depends both on radius and on latitude (for example the tachocline in the solar case). This is the reason why we generalize the formalism, treating the case of a general strong differential rotation, the angular velocity $\Omega$ being a function both of the radius $(r)$ and of the colatitude $(\theta)$, as can be potentially the case in stellar radiation zones during the evolution of stars.

First, we derive the equations ruling the dynamics of waves in a differentially rotating star. Then, we focus on the lowfrequency waves in a differentially rotating stellar radiation zone. We present and discuss the different approximations that can be adopted there, namely the traditional and the JWKB ones, and we derive the associated dynamical equations. Then, we solve them to obtain the spatial structure of the wave pressure fluctuation and velocity field in the quasi-adiabatic approximation (cf. Press 1981; Zahn et al. 1997). A comparison with the weak differential rotation case is presented. Next, we study the induced transports of energy and of angular momentum by waves. We treat the matching of their pressure at the borders with adjacent convective regions where they are excited by turbulent movements. After a short review of the different models that can be adopted for such excitation which rules the waves spectrum, we derive the total transported flux of angular momentum. Finally, the associated dynamical equations governing the evolution of the mean differential rotation on an isobar and its latitudinal fluctuation are obtained, following the formalism used by Mathis \& Zahn (2004) and Mathis \& Zahn (2005). This allows us to treat for the first time the action of a general strong differential rotation on IGWs and their feed-back on the angular momentum transport, which is of major interest for stellar evolution.

In a forthcoming paper (Paper II), this formalism will be applied to the case of solar differential rotation.

\section{Waves in a differentially rotating star}

We have to solve the complete adiabatic inviscid system to treat the wave dynamics in a differentially rotating star. It is formed by the momentum equation

$D_{t} \boldsymbol{V}=-\frac{1}{\rho} \nabla P-\nabla \Phi$

the continuity equation

$D_{t} \rho+\rho \boldsymbol{\nabla} \cdot \boldsymbol{V}=0$,

the equation for the energy, which is given here in the adiabatic limit

$\frac{1}{\Gamma_{1}} D_{t} \ln P-D_{t} \ln \rho=0$

and Poisson's equation for the gravitational potential

$\nabla^{2} \Phi=4 \pi G \rho$.

$D_{t}$ is the Lagrangian derivative: $D_{t}=\partial_{t}+\boldsymbol{V} \cdot \boldsymbol{\nabla} . \boldsymbol{V}$ is the macroscopic velocity field that is the sum of the azimuthal velocity associated with the differential rotation $(\Omega(r, \theta)$ is the angular velocity) and of the wave velocity field, $\boldsymbol{u}$ :

$\boldsymbol{V}(r, \theta, \varphi, t)=r \sin \theta \Omega(r, \theta) \widehat{\mathbf{e}}_{\varphi}+\boldsymbol{u}(r, \theta, \varphi, t)$.

$t$ is the time and $r, \theta, \varphi$ are the usual spherical coordinates with their associated unit vector basis $\left\{\widehat{\boldsymbol{e}}_{k}\right\}_{k=\{r, \theta, \varphi\}}$. In this first step, the meridional circulation that superposes is ignored. $\rho, \Phi$ and $P$ are respectively the density, the gravitational potential and the pressure while $\Gamma_{1}=(\partial \ln P / \partial \ln \rho)_{\mathrm{s}}$ is the adiabatic exponent, $S$ being the macroscopic entropy. $G$ is the universal gravitational constant.

Equations (1-4) are linearized around the differentially rotating steady-state ${ }^{1}$. Each scalar field $X$ (the density, the gravitational potential and the pressure) is expanded as the sum of

\footnotetext{
${ }^{1}$ Here, the angular velocity $\Omega$ is assumed to be time-independant. It will be time-dependant when the angular momentum transport will be considered in Sect. 4. This means that we make a time-scale separation between the wave dynamical one, $t_{\mathrm{d}}=\sigma^{-1}$, and the shortest one which characterizes the transport of angular momentum. In stellar interiors, this is relevant, since in the case of wave-induced transport, this shortest time is of the order of several years (in the Shear Layer Oscillation; see Sect. 4.3.1 and Talon \& Charbonnel 2005) while $t_{\mathrm{d}} \approx 12 \mathrm{~h}$ for $\sigma=1 \mu \mathrm{Hz}$.
} 
its hydrostatic value, $\bar{X}$, and of the wave's associated fluctuation, $\widetilde{X}$, as:

$X(r, \theta, \varphi, t)=\bar{X}(r, \theta)+\widetilde{X}(r, \theta, \varphi, t)$.

We obtain (cf. Unno et al. 1989):

$$
\begin{aligned}
\left(\partial_{t}+\Omega \partial_{\varphi}\right) \boldsymbol{u}+2 \Omega \widehat{\boldsymbol{e}}_{z} \times \boldsymbol{u}+r \sin \theta(\boldsymbol{u} \cdot \boldsymbol{\nabla} \Omega) \widehat{\boldsymbol{e}}_{\varphi} & = \\
& -\frac{1}{\bar{\rho}} \boldsymbol{\nabla} \widetilde{P}-\boldsymbol{\nabla} \widetilde{\Phi}+\frac{\widetilde{\rho}}{\bar{\rho}^{2}} \nabla \bar{P},
\end{aligned}
$$

where $\widehat{\boldsymbol{e}}_{z}=\cos \theta \widehat{\boldsymbol{e}}_{r}-\sin \theta \widehat{\boldsymbol{e}}_{\theta}$ is the unit vector along the rotation axis and $r \sin \theta(\boldsymbol{u} \cdot \boldsymbol{\nabla} \Omega) \widehat{\boldsymbol{e}}_{\varphi}$ is the Coriolis acceleration term due to the differential rotation (see for example Zahn 1966). Here, the centrifugal acceleration $\gamma_{\mathrm{c}}=\frac{1}{2} \Omega^{2} \nabla\left(r^{2} \sin ^{2} \theta\right)$ is ignored (see the discussion at the end of this section).

Next, we have

$$
\begin{aligned}
& \left(\partial_{t}+\Omega \partial_{\varphi}\right) \tilde{\rho}+\boldsymbol{\nabla} \cdot(\bar{\rho} \boldsymbol{u})=0, \\
& \left(\partial_{t}+\Omega \partial_{\varphi}\right)\left(\frac{\widetilde{P}}{\Gamma_{1} \bar{P}}-\frac{\widetilde{\rho}}{\bar{\rho}}\right)+\boldsymbol{u} \cdot\left(\frac{1}{\Gamma_{1}} \nabla \ln \bar{P}-\nabla \ln \bar{\rho}\right)=0
\end{aligned}
$$

and

$\nabla^{2} \widetilde{\Phi}=4 \pi G \widetilde{\rho}$.

The Lagrangian wave's displacement $\boldsymbol{\xi}$ is given by (cf. Unno et al. 1989):

$\boldsymbol{u}=\left(\partial_{t}+\Omega \partial_{\varphi}\right) \boldsymbol{\xi}-r \sin \theta(\boldsymbol{\xi} \cdot \boldsymbol{\nabla} \Omega) \widehat{\boldsymbol{e}}_{\varphi}$.

Next, $\widetilde{X}, \boldsymbol{u}$ and $\xi$ are expanded in a Fourier sery in $\varphi$ and $t$ :

$\widetilde{X}=\sum_{\sigma, m}\left\{X^{\prime}(r, \theta) \exp [\mathrm{i}(m \varphi+\sigma t)]\right\}$,

$\boldsymbol{u}=\sum_{\sigma, m}\left\{\boldsymbol{u}^{\prime}(r, \theta) \exp [\mathrm{i}(m \varphi+\sigma t)]\right\}$

$\xi=\sum_{\sigma, m}\left\{\xi^{\prime}(r, \theta) \exp [\mathrm{i}(m \varphi+\sigma t)]\right\}$,

$\sigma$ being the wave angular velocity in an inertial frame (thus, we assume that the rotation rate at which the waves are generated is zero).

Inserting Eqs. (12), (13) in Eq. (7), the following linearized momentum equation components are obtained:

i $\widehat{\sigma} u_{r}^{\prime}-2 \Omega \sin \theta u_{\varphi}^{\prime}=-\partial_{r} W+\frac{1}{\bar{\rho}^{2}}\left(\rho^{\prime} \partial_{r} \bar{P}-P^{\prime} \partial_{r} \bar{\rho}\right)$,

$\mathrm{i} \widehat{\sigma} u_{\theta}^{\prime}-2 \Omega \cos \theta u_{\varphi}^{\prime}=-\frac{1}{r} \partial_{\theta} W+\frac{1}{\bar{\rho}^{2} r}\left(\rho^{\prime} \partial_{\theta} \bar{P}-P^{\prime} \partial_{\theta} \bar{\rho}\right)$,

$\mathrm{i} \widehat{\sigma} u_{\varphi}^{\prime}+\frac{1}{r \sin \theta}\left(u_{r}^{\prime} \partial_{r}+\frac{u_{\theta}^{\prime}}{r} \partial_{\theta}\right)\left(r^{2} \sin ^{2} \theta \Omega\right)=-\frac{\mathrm{i} m W}{r \sin \theta}$

where

$W=\frac{P^{\prime}}{\bar{\rho}}+\Phi^{\prime}$,

$u_{r}^{\prime}, u_{\theta}^{\prime}, u_{\varphi}^{\prime}$ being respectively the radial, the latitudinal and the azimuthal components of $\boldsymbol{u}^{\prime}$.
Next, the continuity equation (Eq. (8)), the energy equation in the adiabatic limit (Eq. (9)) and Poisson's equation (Eq. (10)) respectively become

$$
\begin{aligned}
\mathrm{i} \widehat{\sigma} \rho^{\prime}+u_{r}^{\prime} \partial_{r} \bar{\rho}+\frac{u_{\theta}^{\prime}}{r} \partial_{\theta} \bar{\rho}+ & \bar{\rho}\left[\frac{1}{r^{2}} \partial_{r}\left(r^{2} u_{r}^{\prime}\right)\right. \\
& \left.+\frac{1}{r \sin \theta} \partial_{\theta}\left(\sin \theta u_{\theta}^{\prime}\right)+\frac{\mathrm{i} m u_{\varphi}^{\prime}}{r \sin \theta}\right]=0 \\
\mathrm{i} \widehat{\sigma}\left(\frac{P^{\prime}}{\Gamma_{1} \bar{P}}-\frac{\rho^{\prime}}{\bar{\rho}}\right)+u_{r}^{\prime}\left(\frac{1}{\Gamma_{1}} \partial_{r} \ln \bar{P}-\partial_{r} \ln \bar{\rho}\right) & \\
+ & \frac{u_{\theta}^{\prime}}{r}\left(\frac{1}{\Gamma_{1}} \partial_{\theta} \ln \bar{P}-\partial_{\theta} \ln \bar{\rho}\right)=0
\end{aligned}
$$

and

$\frac{1}{r^{2}} \partial_{r}\left(r^{2} \partial_{r} \Phi^{\prime}\right)+\frac{1}{r^{2}}\left[\frac{1}{\sin \theta} \partial_{\theta}\left(\sin \theta \partial_{\theta} \Phi^{\prime}\right)-\frac{m^{2}}{\sin ^{2} \theta} \Phi^{\prime}\right]=4 \pi G \rho^{\prime}$.

On the other hand, we get from Eq. (11):

$\xi_{k}^{\prime}=\frac{u_{k}^{\prime}}{\mathrm{i} \widehat{\sigma}}+r \sin \theta\left(\frac{u_{r}^{\prime}}{\mathrm{i} \widehat{\sigma}} \frac{\partial_{r} \Omega}{\mathrm{i} \widehat{\sigma}}+\frac{u_{\theta}^{\prime}}{\mathrm{i} \widehat{\sigma}} \frac{1}{r} \frac{\partial_{\theta} \Omega}{\mathrm{i} \widehat{\sigma}}\right) \varepsilon_{k, \varphi}$,

where $k=\{r, \theta, \varphi\}$ and $\varepsilon_{k, \varphi}=1$ if $k=\varphi$ and 0 otherwise.

In a differentially rotating region, the waves are Dopplerschifted due to the differential rotation. Thus, the local wave angular velocity that corresponds to the operator $\left(\partial_{t}+\Omega \partial_{\varphi}\right)$ is:

$\widehat{\sigma}(r, \theta)=\sigma+m \Omega(r, \theta)$.

This Doppler-shift is an essential ingredient in the angular momentum deposition or extraction respectively through prograde $(m<0)$ and retrograde waves $(m>0)$ damping (see for example in Talon et al. 2002; note that we have chosen here the sign convention adopted by Lee \& Saio 1997; and Mathis et al. 2008).

From now on, we neglect the non-spherical character of the hydrostatic background due to the deformation associated with the centrifugal acceleration, $\gamma_{\mathrm{c}}$. We thus stop the expansion of the equations to the first order in $\varepsilon=\frac{\Omega}{\Omega_{\mathrm{c}}}$ where $\Omega_{\mathrm{c}}=\sqrt{\frac{G M}{R^{3}}}$ is the critical angular velocity of the star, $R$ and $M$ being respectively its radius and its mass. We thus have:

$\bar{X}=\bar{X}(r)$

and the gravity $\bar{g}(r)$ and the Brunt-Väisälä frequency $N(r)$ given by:

$\bar{g}=\frac{\mathrm{d} \bar{\Phi}}{\mathrm{d} r} \quad$ and $\quad N^{2}=\frac{1}{\bar{\rho}} \frac{\mathrm{d} \bar{P}}{\mathrm{~d} r}\left(\frac{\mathrm{d} \ln \bar{\rho}}{\mathrm{d} r}-\frac{1}{\Gamma_{1}} \frac{\mathrm{d} \ln \bar{P}}{\mathrm{~d} r}\right)$.

The general dynamical equations for waves in a differentially rotating star now being given, we focus our attention on radiative regions and on the approximations which can be applied there.

\section{Low-frequency waves in a differentially rotating stellar radiation zone}

\subsection{The traditional approximation}

In the general case, the operator which governs the spatial structure of the waves, the Poincaré operator, is of mixed type (elliptic and hyperbolic) and not separable (for a detailed discussion we refer the reader to Friedlander \& Siegman 1982; Dintrans 1999; Dintrans et al. 1999; and to Dintrans \& Rieutord 2000). This leads to the appearance of detached shear layers associated with 
the underlying singularities of the adiabatic problem that could be crucial for transport and mixing processes in stellar radiation zones, since they are the seat of strong dissipation (Stewartson \& Richard 1969; Stewartson \& Walton 1976; Dintrans et al. 1999; Dintrans \& Rieutord 2000; Ogilvie \& Lin 2004).

However, in the largest part of stellar radiation zones, we are in a regime where $2 \Omega \ll N$. Since we are interested here in lowfrequency waves where $\sigma \ll N$, the traditional approximation, which consists of neglecting the latitudinal component of the rotation vector $\left(\boldsymbol{\Omega}=\Omega(r, \theta) \widehat{\boldsymbol{e}}_{z}=\Omega_{V} \widehat{\boldsymbol{e}}_{r}+\Omega_{H} \widehat{\boldsymbol{e}}_{\theta}\right.$ with $\Omega_{V}=\Omega \cos \theta$ and $\left.\Omega_{H}=-\Omega \sin \theta\right),-\Omega \sin \theta \widehat{\boldsymbol{e}}_{\theta}$, for all latitudes in the momentum equation, can be adopted in the case where $2 \Omega<\sigma$ when $\Omega$ is uniform (see e.g. Eckart 1960; Lindzen \& Chapman 1969; and Miles 1974; for a modern description in a stellar context see Nicholson 1989; Bildsten et al. 1996; Papaloizou \& Savonije 1997; Lee \& Saio 1997; Talon 1997). Then, it has been shown by Friedlander (1987) that variable separation in radial and horizontal eigenfunctions remains possible. This approximation has to be used carefully, as it changes the nature of the Poincaré operator, and removes the singularities and associated shear layers that appear. Therefore, assuming solid-body rotation, it is only valid in the super-inertial regime $2 \Omega<\sigma \ll N$, where the stratification dominates, that corresponds to the ergodic (regular) elliptic gravito-inertial mode family (the $E_{1}$ modes in Dintrans et al. 1999; and Dintrans \& Rieutord 2000). In the sub-inertial regime, where $\sigma \leq 2 \Omega$, that corresponds to the equatorially trapped hyperbolic modes (the $\mathrm{H}_{2}$ modes in Dintrans et al. 1999; and Dintrans \& Rieutord 2000), the traditional approximation fails to reproduce the waves behaviour and the complete momentum equation has to be solved (detailed examples are given in Gerkema \& Shrira 2005 and Gerkema et al. 2007).

Therefore, we restrict ourselves here to the regular lowfrequency waves for which the traditional approximation is usable. Its application domain in the case of general strong differential rotation will be discussed in Sect. 3.4.6.

Let us now adopt a local analysis of the problem in the simplest case of a uniform rotation (see also Lee $\&$ Saio 1997). The wave vector $\boldsymbol{k}$ and Lagrangian displacement $\boldsymbol{\xi}$ are expanded as

$\boldsymbol{k}=k_{V} \widehat{\boldsymbol{e}}_{r}+k_{\theta} \widehat{\boldsymbol{e}}_{\theta}+k_{\varphi} \widehat{\boldsymbol{e}}_{\varphi}=k_{V} \widehat{\boldsymbol{e}}_{r}+\boldsymbol{k}_{H}$

$\boldsymbol{\xi}=\xi_{V} \widehat{\boldsymbol{e}}_{r}+\xi_{\theta} \widehat{\boldsymbol{e}}_{\theta}+\xi_{\varphi} \widehat{\boldsymbol{e}}_{\varphi}=\xi_{V} \widehat{\boldsymbol{e}}_{r}+\boldsymbol{\xi}_{H}$,

where $\boldsymbol{k}_{H}=k_{\theta} \widehat{\boldsymbol{e}}_{\theta}+k_{\varphi} \widehat{\boldsymbol{e}}_{\varphi}, k_{H}=\left|\boldsymbol{k}_{H}\right|, k=|\boldsymbol{k}|, \boldsymbol{\xi}_{H}=\xi_{\theta} \widehat{\boldsymbol{e}}_{\theta}+\xi_{\varphi} \widehat{\boldsymbol{e}}_{\varphi}$, $\xi_{H}=\left|\boldsymbol{\xi}_{H}\right|, \boldsymbol{\xi} \propto \exp [\mathrm{i}(\boldsymbol{k} \cdot \boldsymbol{r}-\sigma t)]$ and $\boldsymbol{\xi}=|\boldsymbol{\xi}|$. writte:

For low-frequency waves in stably stratified regions, we can

$\boldsymbol{k} \cdot \boldsymbol{\xi}=k_{V} \xi_{V}+\boldsymbol{k}_{H} \cdot \boldsymbol{\xi}_{H} \approx 0$,

since $\boldsymbol{\nabla} \cdot(\bar{\rho} \boldsymbol{\xi}) \approx 0$ (this is the anelastic approximation that filters out acoustic waves which have higher frequencies), from which we deduce the following indentity:

$\frac{\xi_{V}}{\xi_{H}} \approx-\frac{k_{H}}{k_{V}}$.

Next, using the results given in Unno et al. (1989), the following dispersion relation for the low-frequency gravito-inertial waves is obtained:

$\sigma^{2} \approx N^{2} \frac{k_{H}^{2}}{k^{2}}+\frac{(2 \boldsymbol{\Omega} \cdot \boldsymbol{k})^{2}}{k^{2}}$.

In this expression, the mixed behaviour of waves is clearly identified, the two terms corresponding respectively to the dispersion relations of IGWs, $\sigma^{2} \approx N^{2} \frac{k_{H}^{2}}{k^{2}}$, and of inertial waves,

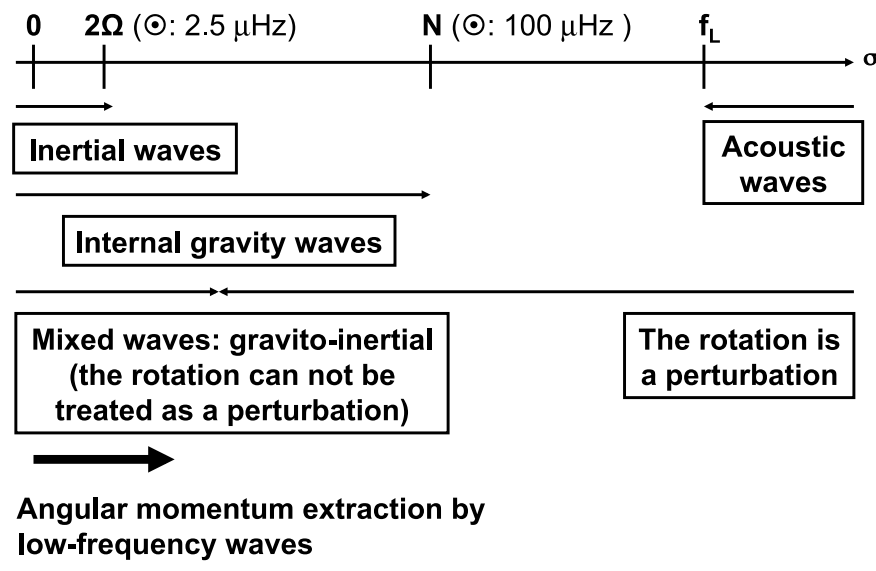

Fig. 1. Wave types in differentially rotating stellar radiation zone and associated frequencies (where $f_{\mathrm{L}}$ is the Lamb's frequency).

$\sigma^{2} \approx \frac{(2 \Omega \cdot k)^{2}}{k^{2}}$. In the case where the "traditional" frequency hierarchy, $2 \Omega \ll N$ and $\sigma \ll N$, is verified (this is the case for example in the radiative region of the Sun, cf. Fig. 1), the previous dispersion relation gives:

$\frac{k_{H}^{2}}{k^{2}} \ll 1$

The vertical wave vector is then larger than the horizontal one while the displacement vector is almost horizontal:

$\left|k_{H}\right| \ll\left|k_{V}\right|, \quad\left|\xi_{V}\right| \ll\left|\xi_{H}\right|$.

On the other hand, we get $(2 \boldsymbol{\Omega} \cdot \boldsymbol{k}) \approx 2 \Omega_{V} k_{V}$. The latitudinal component of the rotation vector can thus be neglected in the whole sphere.

\subsection{The JWKB approximation}

Under the assumption that $\sigma \ll N$, each scalar field and each component of $\boldsymbol{u}^{\prime}$ can be expanded using the JWKB approximation (see Landau \& Lifchitz 1966; Fröman \& Fröman 1965; Vallée \& Soares 1998, and references therein for mathematical details). In this case, the vertical wave number is very large, the associated wave-length being thus very small. Therefore, the spatial variation of the wave is very rapid compared to that of the hydrostatic background (cf. compared to those of $\bar{\rho}, \bar{g}$ and $\bar{P}$ ). Then, the wave spatial structure can be described by the product of a plane-like wave function multiplied by a slowly varying envelope and we obtain:

$u_{k}^{\prime}=\widehat{u}_{k}(r, \theta) \mathcal{S}(r)$,

$\xi_{k}^{\prime}=\widehat{\xi}_{k}(r, \theta) \mathcal{S}(r)$

with

$\widehat{\xi}_{k}=\frac{\widehat{u}_{k}}{\mathrm{i} \widehat{\sigma}}+r \sin \theta\left(\frac{\widehat{u}_{r}}{\mathrm{i} \widehat{\sigma}} \frac{\partial_{r} \Omega}{\mathrm{i} \widehat{\sigma}}+\frac{\widehat{u}_{\theta}}{\mathrm{i} \widehat{\sigma}} \frac{1}{r} \frac{\partial_{\theta} \Omega}{\mathrm{i} \widehat{\sigma}}\right) \varepsilon_{k, \varphi}$,

$\rho^{\prime}=\widehat{\rho}(r, \theta) \mathcal{S}(r)$,

$P^{\prime}=\widehat{P}(r, \theta) \mathcal{S}(r)$

and

$\Phi^{\prime}=\Phi_{\mathrm{CZ}}(r, \theta)+\widehat{\Phi}(r, \theta) \mathcal{S}(r)$.

The case of the fluctuation of the gravitational potential is particular since it is the sum of the fluctuating potential associated 
with the waves propagating in the studied radiation zone and of the one associated with the movements in the convection zone, $\Phi_{\mathrm{CZ}}$.

The JWKB phase function is given by:

$\mathcal{S}(r)=\exp \left[\mathrm{i}\left(\int_{r}^{r_{\mathrm{c}}} k_{V}\left(r^{\prime}\right) \mathrm{d} r^{\prime}+\frac{\pi}{2}\right)\right]$,

where the property of low-frequency waves given in Eq. (32) has been used to neglect $\boldsymbol{k}_{H}$ in $\exp \left[\mathrm{i} \int_{\boldsymbol{r}} \boldsymbol{k} \cdot \mathrm{d} \boldsymbol{r}^{\prime}\right]$. The arbitrary phase origin is chosen so that $\xi_{r}^{\prime}$ is real at $r=r_{\mathrm{c}}$ (see Zahn et al. 1997; Mathis 2005; Pantillon et al. 2007; and Mathis et al. 2008). Moreover, the JWKB amplitude of the fluctuation $\mathcal{A}(r)=A \frac{1}{k_{V}^{1 / 2}}$ is absorbed in the $\widehat{f}(r, \theta)$ functions.

If the JWKB approximation is adopted, this also implies that the quasi-linear approximation, where the non-linear wavewave interactions are neglected, is assumed.

Internal gravity waves - induced transport in stellar interiors was first studied by Press (1981). In this work, he emphasizes the possible non-linearity of the problem of IGWs excited by turbulent convective movements. He then shows that JWKB solutions, using crude prescriptions for the wave excitation, are at the limit between the linear and the non-linear regime. Furthermore, Rogers et al. (2008) obtain results where the non-linear regime develops in the case of an excited spectrum at the convectionradiation border computed through 2-D numerical simulations which account for a real solar stratification (see Sect. 4.2.1 for a more detailed description). This non-linear behaviour then shows that the quasi-linear approximation has to be used carefully depending on the excited spectrum that is assumed.

As discussed by Rogers et al. (2008), the quasi-linear approximation is relevant as long as the Froude number $\left(F_{r}\right)$, which gives the ratio between the wave-inertia term and the stratification one, is small compared to unity. This number has been computed by Rogers \& Glatzmaier (2006; cf. Fig. 4 in this paper) in the solar case using the same numerical simulations as those discussed above. Then, they showed that $F_{\mathrm{r}} \ll 1$ in the bulk of the radiation zone, while it strongly grows in the tachocline where IGWs are excited by the turbulent convection and at the center because of the wave's geometrical focusing already identified by Press (1981).

Therefore, it is reasonable to adopt the quasi-linear approximation, being aware that it has to be used with caution in the excitation region and at the center.

\subsection{Dynamical equations}

Simultaneously using the traditional and the JWKB approximations and assuming the anelastic one where sonic waves are filtered (i.e. $\boldsymbol{\nabla} \cdot(\bar{\rho} \boldsymbol{u}) \approx 0$ ), we derive the dynamical equations for low-frequency waves in differentially rotating radiation zones. Substituting the expansion given in Eq. (33) to Eqs. (15-17), the final radial, latitudinal and azimuthal components of the momentum equation are obtained:

$\mathrm{i} \widehat{\sigma} \widehat{u}_{r}=\mathrm{i} k_{V} \widehat{W}-\frac{\widehat{\rho}}{\bar{\rho}} \bar{g}$,

$\mathrm{i} \widehat{\sigma} \widehat{u}_{\theta}-2 \Omega \cos \theta \widehat{u}_{\varphi}=-\frac{1}{r} \partial_{\theta} \widehat{W}$,

$\mathrm{i} \widehat{\sigma} \widehat{u}_{\varphi}+\frac{\widehat{u}_{\theta}}{\sin \theta} \partial_{\theta}\left(\sin ^{2} \theta \Omega\right)=-\frac{\mathrm{i} m \widehat{W}}{r \sin \theta}$, where

$\widehat{W}=\frac{\widehat{P}}{\bar{\rho}}$.

The different simplifications adopted for each component of the momentum equation have to be detailed.

For its radial component (Eq. (15)), the traditional approximation, for which it is assumed that $2 \Omega \ll N$, allows one to neglect the radial component of the Coriolis acceleration which is thus strongly dominated in the vertical direction by the buoyancy restoring force. Furthermore, in a rigourous way, the inertial term i $\widehat{\sigma} \widehat{u}_{r}$ also has to be neglected since $\sigma \ll N$. However, it is first conserved here to make the historical link with the works in the non-rotating case by Press (1981), Schatzman (1993) and Zahn et al. (1997) and with those in the uniformly rotating case by Pantillon et al. (2007) and Mathis et al. (2008) where it is conserved. Finally, the last right hand-side term $-1 / \bar{\rho}^{2} \partial_{r} \bar{\rho} P^{\prime}$ is not taken into account because of the anelastic approximation. Then, the latitudinal component (Eq. (16)) simplifies since $\partial_{\theta} \bar{P}=\partial_{\theta} \bar{\rho}=0$, the other terms all being of the same order of magnitude and thus conserved. Finally, the term $1 /(r \sin \theta) \widehat{u}_{r} \partial_{r}\left(r^{2} \sin ^{2} \theta \Omega\right)$ is neglected in the azimuthal component (Eq. (17)) since the wave's Lagrangian displacement is mostly horizontal (and thus $\widehat{u}_{r} \ll \widehat{u}_{\theta}$ ) for low-frequency IGWs (cf. Eq. (32) and the discussion in Sect. 3.4.5).

In addition, the continuity equation is ${ }^{2}$ :

$-\mathrm{i} k_{V} \widehat{u}_{r}+\frac{1}{r \sin \theta} \partial_{\theta}\left(\sin \theta \widehat{u}_{\theta}\right)+\frac{\mathrm{i} m \widehat{u}_{\varphi}}{r \sin \theta}=0$

while the energy equation becomes

$-\mathrm{i} \widehat{\sigma} \frac{\widehat{\rho}}{\bar{\rho}}+\frac{N^{2}}{\bar{g}} \widehat{u}_{r}=0$.

Finally, Poisson's equation is given by:

$-k_{V}^{2} \widehat{\phi}=4 \pi G \widehat{\rho}$.

Cowling's approximation, in which the fluctuation of the gravitational potential is neglected in the momentum equation, is made (Cowling 1941). Therefore, $\widehat{W}$ does not involve $\widehat{\phi}$, the wave selfgravitation.

We are now ready to derive the wave spatial structure.

\subsection{Spatial structure of the velocity field and associated properties}

\subsubsection{Spatial structure of the horizontal components of the velocity}

The first step is to derive the spatial structure of the horizontal components of the velocity field as a function of $\widehat{W}=\widehat{P} / \bar{\rho}$. To achieve this, we succesively eliminate $\widehat{u}_{\theta}$ and $\widehat{u}_{\varphi}$ between Eqs. (41) and (42). This leads to the following expressions for each of them:

$\widehat{u}_{\theta}=\frac{\mathrm{i}}{r \widehat{\sigma}} \frac{1}{\mathcal{D}(r, \theta ; \widehat{v})}\left[\partial_{\theta} \widehat{W}-\mathrm{i} \widehat{v} \frac{\cos \theta}{\sin \theta}(\mathrm{i} m \widehat{W})\right]$,

$\widehat{u}_{\varphi}=\frac{\mathrm{i}}{r \widehat{\sigma}} \frac{1}{\mathcal{D}(r, \theta ; \widehat{v})}\left[\mathrm{i}\left(\widehat{v} \cos \theta+\frac{\partial_{\theta} \Omega}{\widehat{\sigma}} \sin \theta\right) \partial_{\theta} \widehat{W}+\frac{\mathrm{i} m \widehat{W}}{\sin \theta}\right]$.

\footnotetext{
${ }^{2}$ Since the JWKB approximation is adopted, we keep only the highest order derivative term in the radial direction. Then, the one associated with $\partial_{r} \bar{\rho}$ is neglected.
} 
We have defined the local spin parameter $\widehat{v}(r, \theta)$, which is the inverse of the local Rossby number, namely the ratio of the local inertial frequency to the wave's local frequency:

$\widehat{v}(r, \theta)=\frac{2 \Omega(r, \theta)}{\widehat{\sigma}(r, \theta)}$

and $\mathcal{D}$ that depends on the rotation rate $(\Omega)$ and on its latitudinal gradient $^{3}$ :

$\mathcal{D}(r, \theta ; \widehat{v})=1-\widehat{v}^{2} \cos ^{2} \theta-\widehat{v} \frac{\partial_{\theta} \Omega}{\widehat{\sigma}} \cos \theta \sin \theta$.

Here, following Ogilvie \& Lin (2004), we notice that in the case where $\widehat{\sigma}=0$ we get the corotation resonance while the case where $\mathcal{D}=0$ is equivalent to the Lindblad resonance encountered in accretion discs (see for example Goldreich \& Tremaine 1979).

We make the hypothesis, which will be verified in the next section, that $\widehat{W}$ can be expanded as

$\widehat{W}=\sum_{j} w_{j, m}(r, x ; \widehat{v})$

where $w_{j, m}$ is its $j$ th spectral component and $x=\cos \theta$.

Then, it comes from Eq. (47) that:

$\widehat{u}_{\theta}=\sum_{j} \widehat{u}_{\theta ; j, m}(r, x ; \widehat{v})$

where the $j$ th spectral component is given by:

$\widehat{u}_{\theta ; j, m}=\mathrm{i} \frac{1}{r} \widehat{\sigma} G_{j, m}^{\theta}(r, x ; \widehat{v})$

with

$\mathcal{G}_{j, m}^{\theta}(r, x ; \widehat{v})=O_{\widehat{v} ; m}^{\theta}\left[w_{j, m}(r, x ; \widehat{v})\right]$,

the linear operator $O_{\widehat{v} ; m}^{\theta}$ being:

$O_{\widehat{v} ; m}^{\theta}=\frac{1}{\widehat{\sigma}^{2}} \frac{1}{\mathcal{D}(r, x ; \widehat{v}) \sqrt{1-x^{2}}}\left[-\left(1-x^{2}\right) \frac{\mathrm{d}}{\mathrm{d} x}+m \widehat{v} x\right]$

where

$\mathcal{D}(r, x ; \widehat{v})=1-\widehat{v}^{2} x^{2}+\widehat{v} \frac{\partial_{x} \Omega}{\widehat{\sigma}} x\left(1-x^{2}\right)$.

Similarly, we obtain from Eq. (48):

$\widehat{u}_{\varphi}=\sum_{j} \widehat{u}_{\varphi ; j, m}(r, x ; \widehat{v})$

with

$\widehat{u}_{\varphi ; j, m}=-\frac{1}{r} \widehat{\sigma}^{\varphi}{ }_{j, m}(r, x ; \widehat{v})$

where

$\mathcal{G}_{j, m}^{\varphi}(r, x ; \widehat{v})=O_{\widehat{v} ; m}^{\varphi}\left[w_{j, m}(r, x ; \widehat{v})\right]$,

3 It can also be written as:

$$
\mathcal{D}(r, \theta ; \widehat{v})=1-\widehat{v}^{2}\left[\cos ^{2} \theta+\frac{1}{2} \partial_{\theta}(\ln \Omega) \cos \theta \sin \theta\right] .
$$

the linear operator $O_{\widehat{v} ; m}^{\varphi}$ being given by:

$$
\begin{aligned}
O_{\widehat{v} ; m}^{\varphi}= & \frac{1}{\widehat{\sigma}^{2}} \frac{1}{\mathcal{D}(r, x ; \widehat{v}) \sqrt{1-x^{2}}} \\
& \times\left[-\left(\widehat{v} x-\left(1-x^{2}\right) \frac{\partial_{x} \Omega}{\widehat{\sigma}}\right)\left(1-x^{2}\right) \frac{\mathrm{d}}{\mathrm{d} x}+m\right] .
\end{aligned}
$$

From the expressions obtained for $O_{\widehat{v} ; m}^{\theta}$ and $O_{\widehat{v} ; m}^{\varphi}$, one can note the dependance of $\widehat{u}_{\theta}$ and $\widehat{u}_{\varphi}$ on the differential rotation profile given by $\Omega(r, \theta)$.

The spatial structure of the latitudinal and azimuthal components of the velocity field is now derived as a function of the pressure field. We have to derive its governing equation.

\subsubsection{Spatial structure of the radial component of the velocity field and of the pressure}

Eliminating the wave's density fluctuation $\widehat{\rho}$ between the radial momentum equation (Eq. (40)) and the energy equation (Eq. (45)), we obtain the relation between $\widehat{u}_{r}$ and $\widehat{W}$ :

$\widehat{u}_{r}=-\left(\frac{N^{2}}{\widehat{\sigma}^{2}}-1\right)^{-1} \frac{k_{V}}{\widehat{\sigma}} \widehat{W} \approx-\frac{\widehat{\sigma}}{N^{2}} k_{V} \widehat{W}$.

Inserting it in the continuity equation (Eq. (44)), we get for $w_{j, m}$, as defined in Eq. (51):

$$
\begin{aligned}
O_{\widehat{v} ; m}\left[w_{j, m}(r, x ; \widehat{v})\right] & =-\frac{k_{V}^{2} r^{2}}{\widehat{\sigma}^{2}}\left(\frac{N^{2}}{\widehat{\sigma}^{2}}-1\right)^{-1} w_{j, m}(r, x ; \widehat{v}) \\
& \approx-\frac{k_{V}^{2} r^{2}}{N^{2}} w_{j, m}(r, x ; \widehat{v}),
\end{aligned}
$$

where the "Generalized Laplace Operator" (hereafter GLO), $O_{\bar{v} ; m}$, is derived:

$$
\begin{aligned}
O_{\widehat{v} ; m}= & \frac{1}{\widehat{\sigma}} \frac{\mathrm{d}}{\mathrm{d} x}\left[\frac{\left(1-x^{2}\right)}{\widehat{\sigma} \mathcal{D}(r, x ; \widehat{v})} \frac{\mathrm{d}}{\mathrm{d} x}\right]-\frac{m}{\widehat{\sigma}^{2} \mathcal{D}(r, x ; \widehat{v})}\left(1-x^{2}\right) \frac{\partial_{x} \Omega}{\widehat{\sigma}} \frac{\mathrm{d}}{\mathrm{d} x} \\
& -\frac{1}{\widehat{\sigma}}\left[\frac{m^{2}}{\widehat{\sigma} \mathcal{D}(r, x ; \widehat{v})\left(1-x^{2}\right)}+m \frac{\mathrm{d}}{\mathrm{d} x}\left(\frac{\widehat{v} x}{\widehat{\sigma} \mathcal{D}(r, x ; \widehat{v})}\right)\right] \cdot(63)
\end{aligned}
$$

$O_{\bar{v} ; m}$ is called the GLO since it reduces to the classical Laplace tidal operator in the case of a uniform rotation $\Omega(r, \theta)=\bar{\Omega}_{\mathrm{s}}, \bar{\Omega}_{\mathrm{s}}$ being the considered solid-body angular velocity (see Laplace 1799; and the detailed discussion in Sect. 3.4.5).

The $w_{j, m}$ are thus the eigenfunctions of the GLO, namely

$O_{\widehat{v} ; m}\left[w_{j, m}(r, x ; \widehat{v})\right]=-\lambda_{j, m}(r ; \widehat{v}) w_{j, m}(r, x ; \widehat{v})$,

the eigenvalues $\lambda_{j, m}$ being deduced from the following dispersion relation ${ }^{4}$ :

$k_{V ; j, m}^{2}(r)=\frac{\lambda_{j, m}(r ; \widehat{v}) N^{2}}{r^{2}}$

that determines the radial wave number $k_{V ; j, m}$ at each $r$. The $\lambda_{j, m}>0$ solutions correspond to the propagating waves, namely the gravito-inertial waves, while the $\lambda_{j, m}<0$ solutions correspond to the evanescent ones.

\footnotetext{
${ }_{4} \lambda_{j, m}$ has the dimension of a times squared $\left[t^{2}\right]$ and is thus expressed in $s^{2}$ (see also Eq. (83)).
} 
Following Ogilvie \& Lin (2004), it can be shown that the GLO is self-adjoint, namely that

$\int_{-1}^{1} f^{*}(x) \mathcal{O}_{\bar{v}, m}[g(x)] \mathrm{d} x=\left[\int_{-1}^{1} g^{*}(x) \mathcal{O}_{\bar{v} ; m}[f(x)] \mathrm{d} x\right]^{*}$

for any two functions $f$ and $g$ satisfying the regularity conditions at the poles ( ${ }^{*}$ is the complex conjugaison). The eigenvalues $\lambda_{j, m}$ are therefore real while the eigenfunctions corresponding to distinct eigenvalues are orthogonal with:

$$
\int_{-1}^{1} w_{i, m}^{*}(r, x ; \widehat{v}) w_{j, m}(r, x ; \widehat{v}) \mathrm{d} x=C_{i, m} \delta_{i, j},
$$

where $\delta_{i, j}$ is the classical Kronecker symbol and $C_{i, m}$ the normalization factor.

It can be shown that the sign of $\lambda_{j, m}$ depends on that of $\mathcal{D}$; see Ogilvie \& Lin (2004) for a more detailed discussion. Singular points of Eq. (64) occur at the poles, or when $\widehat{\sigma}$ or $\mathcal{D}$ vanish (these are respectively the corotation resonance or the Lindblad one, as has been discussed).

The boundary conditions have been given by Ogilvie \& Lin (2004): close to the north pole, the two independent solutions are $w_{j, m} \propto \theta^{m}$ and $w_{j, m} \propto \theta^{-m}$ in the case where $m \neq 0$ while we get $w \propto 1$ or $w \propto \ln \theta$ when $m=0$. The condition that the $w_{j, m}$ are bounded selects the regular solution. A similar condition is required at the south pole. This provides the two boundary conditions for our eigenvalue problem.

Moreover, as it can be seen immediately, eigenvalues depend on the radial coordinate, $r$. Ogilvie \& Lin (2004) describe a "parametric" dependance, since $O_{\bar{v}, m}$ is a differential operator in $x$ only. We prefer here to say that the use of the JWKB solution allows us to reduce the problem of a bidimensionnal partial differential equation in $r$ and $\theta$ to the one of a differential equation in $x$ for each $r$, the obtained solution being also completely 2D without any variable separation in $r$ and $x$. This is because the GLO depends on $x$, but also on $r$ through $\widehat{v}$ and $\mathcal{D}$. In this way, even in the case of a shellular rotation $\Omega(r, \theta)=\bar{\Omega}(r)$, the problem is not separable, the only separable case being the weak differential rotation one (see Sect. 3.4.5).

\subsubsection{Phase and group velocities; radiative damping}

Using the wave's dispersion relation given in Eq. (65), the monochromatic vertical group velocity is derived

$V_{g ; j, m}^{V}=\frac{\mathrm{d} \widehat{\sigma}}{\mathrm{d} k_{V ; j, m}}=-\frac{\widehat{\sigma}}{k_{V ; j, m}}=-V_{p ; j, m}^{V}<0$,

$V_{p ; j, m}^{V}$ being the associated vertical phase velocity; it is negative here since we are studying the case of a solar-type star where waves are excited by the turbulent convection in an upper external envelope (the opposite is obtained in the case of massive stars where waves are excited by a convective core).

Moreover, the vertical radiative damping (we have to remember that $k_{V ; j, m} \gg k_{H ; j, m}$ ) is given by (see for example Kumar et al. 1999):

$\tau_{j, m}(r, \theta ; \widehat{v})=\int_{r}^{r_{\mathrm{c}}} \frac{K k_{V ; j, m}^{2}}{\left|V_{g ; j, m}\right|} \mathrm{d} r^{\prime}=\int_{r}^{r_{\mathrm{c}}} K \frac{\lambda_{j, m}^{3 / 2}(r ; \widehat{v}) N^{3}}{\widehat{\sigma}} \frac{\mathrm{d} r^{\prime}}{r^{\prime 3}}$,

$K$ being the thermal diffusivity. One can note that the nonuniform rotation modifies the damping rate since $\lambda_{j, m}$ is now in the integrand of the dissipation integral (cf. Eq. (91)). Moreover, since $O_{\bar{v} ; m}$ depends on $m$, the differential damping between the prograde and the retrograde waves is modified due to the action of the Coriolis acceleration as in the weak differential rotation case (see Mathis 2005; Pantillon et al. 2007; and Mathis et al. 2008).

As in Press (1981) and Zahn et al. (1997), we adopt here the quasi-adiabatic approximation. In this way, the pressure fluctuation and the velocity field of a monochromatic wave are given by:

$P_{j, m}(\boldsymbol{r}, t)=P_{j, m}^{\mathrm{Ad}}(\boldsymbol{r}, t) \exp \left[-\tau_{j, m}(\boldsymbol{r}) / 2\right]$,

$\boldsymbol{u}_{j, m}(\boldsymbol{r}, t)=\boldsymbol{u}_{j, m}^{\mathrm{Ad}}(\boldsymbol{r}, t) \exp \left[-\tau_{j, m}(\boldsymbol{r}) / 2\right]$,

$P_{j, m}^{\mathrm{Ad}}$ and $\boldsymbol{u}_{j, m}^{\mathrm{Ad}}$ being their respective spatial structure in the adiabatic case.

\subsubsection{Final pressure field and velocity field}

Using the results reported previously, the pressure field $\widetilde{P}$ and the velocity field $\boldsymbol{u}$ of the low-frequency waves in a differentially rotating radiation zone can be derived. Assuming the quasiadiabatic approximation, we obtain for the pressure field:

$\widetilde{P}(r, \theta, \varphi, t)=\sum_{\sigma, m, j} P_{j, m}^{\prime}(r, \theta, \varphi, t)$,

where

$$
\begin{aligned}
P_{j, m}^{\prime}(r, \theta, \varphi, t)= & -\bar{\rho} w_{j, m}(r, \theta ; \widehat{v}) \sin \left[\Phi_{j, m}(r, \varphi, t)\right] \\
& \times \exp \left[-\tau_{j, m}(r, \theta ; \widehat{v}) / 2\right],
\end{aligned}
$$

the phase function $\Phi_{j, m}$ being given by:

$\Phi_{j, m}(r, \varphi, t)=\sigma t+\int_{r}^{r_{\mathrm{c}}} k_{V ; j, m} \mathrm{~d} r^{\prime}+m \varphi$.

Then, we get for the velocity field:

$\boldsymbol{u}=\sum_{k=\{r, \theta, \varphi\}}\left[\sum_{\sigma, m, j} u_{k ; j, m}(r, \theta, \varphi, t)\right] \widehat{\boldsymbol{e}}_{k}$

where

$$
\begin{aligned}
u_{r ; j, m}(r, \theta, \varphi, t)= & \frac{\widehat{\sigma}}{N} \frac{\lambda_{j, m}^{1 / 2}(r ; \widehat{v})}{r} w_{j, m}(r, \theta ; \widehat{v}) \sin \left[\Phi_{j, m}(r, \varphi, t)\right] \\
& \times \exp \left[-\tau_{j, m}(r, \theta ; \widehat{v}) / 2\right]
\end{aligned}
$$

$$
\begin{aligned}
u_{\theta ; j, m}(r, \theta, \varphi, t)= & -\frac{\widehat{\sigma}}{r} \mathcal{G}_{j, m}^{\theta}(r, \theta ; \widehat{v}) \cos \left[\Phi_{j, m}(r, \varphi, t)\right] \\
& \times \exp \left[-\tau_{j, m}(r, \theta ; \widehat{v}) / 2\right],
\end{aligned}
$$

$$
\begin{aligned}
u_{\varphi ; j, m}(r, \theta, \varphi, t)= & \frac{\widehat{\sigma}}{r} \mathcal{G}_{j, m}^{\varphi}(r, \theta ; \widehat{v}) \sin \left[\Phi_{j, m}(r, \varphi, t)\right] \\
& \times \exp \left[-\tau_{j, m}(r, \theta ; \widehat{v}) / 2\right]
\end{aligned}
$$

On the other hand, since we have to use it to compute the vertical flux of angular momentum (see Sect. 4.1.2, Bretherton 1969; and Pantillon et al. 2007), we derive from Eq. (11):

$\xi_{\theta}=\sum_{\sigma, m, j} \xi_{\theta ; j, m}(r, \theta, \varphi, t)$

with

$$
\begin{aligned}
\xi_{\theta ; j, m}(r, \theta, \varphi, t)= & -\frac{1}{r} \mathcal{G}_{j, m}^{\theta}(r, \theta ; \widehat{v}) \sin \left[\Phi_{j, m}(r, \varphi, t)\right] \\
& \times \exp \left[-\tau_{j, m}(r, \theta ; \widehat{v}) / 2\right] .
\end{aligned}
$$




\subsubsection{Discussion of the weak differential rotation case}

In the weak differential rotation case where the angular velocity is expanded such that

$\Omega(r, \theta)=\bar{\Omega}_{\mathrm{s}}+\delta \bar{\Omega}(r)$

with $\delta \bar{\Omega} \ll \bar{\Omega}_{\mathrm{s}}$, the structure of low-frequency waves is mainly modified by the solid-body rotation, $\bar{\Omega}_{\mathrm{s}}$, the residual radial differential rotation, $\delta \bar{\Omega}$, being only taken into account in the radiative damping term (this is the case treated by Mathis 2005; Pantillon et al. 2007; and Mathis et al. 2008). In this case, the local frequency and the local spin parameter become

$\widehat{\sigma}=\widetilde{\sigma}=\sigma_{\mathrm{s}}+m \delta \bar{\Omega}$ and $\widehat{v}_{\mathrm{s}}=v_{\mathrm{s}}=\frac{2 \bar{\Omega}_{\mathrm{s}}}{\sigma_{\mathrm{s}}}$,

where $\sigma_{\mathrm{s}}=\sigma+m \bar{\Omega}_{\mathrm{s}}$.

The GLO, $O_{\bar{v}_{\mathrm{s}} ; m}$, then reduces to the classical Laplace's tidal operator $\mathcal{L}_{v_{s} ; m}$ :

$$
\begin{aligned}
& O_{\widehat{v}_{\mathrm{s}} ; m}=\frac{1}{\sigma_{\mathrm{s}}^{2}} \mathcal{L}_{v_{\mathrm{s}} ; m} \\
& =\frac{1}{\sigma_{\mathrm{s}}^{2}}\left[\frac{\mathrm{d}}{\mathrm{d} x}\left(\frac{1-x^{2}}{1-v_{\mathrm{s}}^{2} x^{2}} \frac{\mathrm{d}}{\mathrm{d} x}\right)-\frac{1}{1-v_{\mathrm{s}}^{2} x^{2}}\left(\frac{m^{2}}{1-x^{2}}+m v_{\mathrm{s}} \frac{1+v_{\mathrm{s}}^{2} x^{2}}{1-v_{\mathrm{s}}^{2} x^{2}}\right)\right]
\end{aligned}
$$

of which the eigenfunctions are the usual Hough's functions $\Theta_{j, m}\left(x ; v_{\mathrm{s}}\right)$ (Hough 1898; Longuet-Higgins 1968; Miles 1977) that depend on $x$ only since $v_{\mathrm{S}}$ is now uniform in the considered radiation zone, $\mathcal{L}_{v_{\mathrm{s}} ; m}$ being thus a linear differential operator in $x$ only.

The dispersion relation obtained in Eq. (65) is then given by

$k_{V ; j, m}^{2}(r)=\frac{N^{2}}{\sigma_{\mathrm{s}}^{2}} \frac{\Lambda_{j, m}\left(v_{\mathrm{s}}\right)}{r^{2}} \quad$ with $\quad \Lambda_{j, m}\left(v_{\mathrm{s}}\right)=\sigma_{\mathrm{s}}^{2} \lambda_{j, m}\left(\widehat{v}_{\mathrm{s}}\right)$,

where the classical eigenvalues for the Laplace tidal operator, $\Lambda_{j, m}\left(v_{\mathrm{s}}\right)$, have been introduced and related to the $\lambda_{j, m}\left(\widehat{v}_{\mathrm{s}}\right)$.

Finally, the operators $O_{\widehat{v}_{s} ; m}^{\theta}$ and $O_{\widehat{v}_{s} ; m}^{\varphi}$ are simplified

$$
\begin{aligned}
O_{\widehat{v}_{\mathrm{s}} ; m}^{\theta} & =\frac{1}{\sigma_{\mathrm{s}}^{2}} \mathcal{L}_{v_{\mathrm{s}} ; m}^{\theta} \\
& =\frac{1}{\sigma_{\mathrm{s}}^{2}} \times\left[\frac{1}{\left(1-v_{\mathrm{s}}^{2} x^{2}\right) \sqrt{1-x^{2}}}\left[-\left(1-x^{2}\right) \frac{\mathrm{d}}{\mathrm{d} x}+m v_{\mathrm{s}} x\right]\right] \\
O_{\widehat{v}_{\mathrm{s}} ; m}^{\varphi} & =\frac{1}{\sigma_{\mathrm{s}}^{2}} \mathcal{L}_{v_{\mathrm{s}} ; m}^{\varphi} \\
& =\frac{1}{\sigma_{\mathrm{s}}^{2}} \times\left[\frac{1}{\left(1-v_{\mathrm{s}}^{2} x^{2}\right) \sqrt{1-x^{2}}}\left[-v_{\mathrm{s}} x\left(1-x^{2}\right) \frac{\mathrm{d}}{\mathrm{d} x}+m\right],\right.
\end{aligned}
$$

where the linear differential operators $\mathcal{L}_{v_{\mathrm{s}} ; m}^{\theta}$ and $\mathcal{L}_{\nu_{\mathrm{s}} ; m}^{\varphi}$ have been defined in Mathis (2005) and in Pantillon et al. (2007).

We thus obtain a separation of variables in $r$ and $\theta$, as in Lee \& Saio (1997), Mathis (2005), Pantillon et al. (2007) and Mathis et al. (2008), with in the adiabatic case:

$w_{j, m}(r, \theta ; \widehat{v})=\frac{P_{j, m}(r)}{\bar{\rho}} \Theta_{j, m}\left(\cos \theta ; v_{\mathrm{s}}\right)$, and

$$
\begin{aligned}
& \mathcal{G}_{j, m}^{\theta}(r, \theta ; \widehat{v})=\frac{1}{\sigma_{\mathrm{s}}^{2}} \frac{P_{j, m}(r)}{\bar{\rho}} \mathcal{H}_{j, m}^{\theta}\left(\cos \theta ; v_{\mathrm{s}}\right), \\
& \mathcal{G}_{j, m}^{\varphi}(r, \theta ; \widehat{v})=\frac{1}{\sigma_{\mathrm{s}}^{2}} \frac{P_{j, m}(r)}{\bar{\rho}} \mathcal{H}_{j, m}^{\varphi}\left(\cos \theta ; v_{\mathrm{s}}\right),
\end{aligned}
$$

where we recall the respective definition of $\mathcal{H}_{j, m}^{\theta}$ and of $\mathcal{H}_{j, m}^{\varphi}$ :

$\mathcal{H}_{j, m}^{\theta}\left(x ; v_{\mathrm{s}}\right)=\mathcal{L}_{v_{\mathrm{s}} ; m}^{\theta}\left[\Theta_{j, m}\left(x ; v_{\mathrm{s}}\right)\right]$

$\mathcal{H}_{j, m}^{\varphi}\left(x ; v_{\mathrm{s}}\right)=\mathcal{L}_{v_{\mathrm{s}} ; m}^{\varphi}\left[\Theta_{j, m}\left(x ; v_{\mathrm{s}}\right)\right]$.

Finaly, the thermal damping rate becomes using Eq. (83):

$\tau_{j, m}(r, \theta ; \widehat{v})=\Lambda_{j, m}^{3 / 2}\left(v_{\mathrm{s}}\right) \int_{r}^{r_{\mathrm{c}}} K \frac{N^{3}}{\tilde{\sigma}^{4}} \frac{\mathrm{d} r^{\prime}}{r^{\prime 3}}$

\subsubsection{The traditional approximation in the case of general differential rotation}

In the weak differential rotation case (see Sect. 3.4.5), the traditional approximation can be applied in spherical shell(s) where

$$
\begin{aligned}
\mathcal{D}\left(r, x ; v_{\mathrm{s}}\right)=1-v_{\mathrm{s}}^{2} \cos ^{2} \theta>0 \quad & \text { everywhere } \\
& (\forall r \text { and } \forall \theta \in[0, \pi]),
\end{aligned}
$$

thus as long as $2 \bar{\Omega}_{\mathrm{s}}<\sigma_{\mathrm{s}} \ll N\left(v_{\mathrm{s}}<1\right)$ (cf. Figs. 2 and 3), that corresponds to the super-inertial regime where the adiabatic wave operator is elliptic and to regular (elliptic) gravito-inertial waves (see Dintrans \& Rieutord 2000, for a detailed classification of gravito-inertial waves). In the other spherical shell(s), where both $\mathcal{D} \leq 0$ and $\mathcal{D}>0$, which corresponds to the subinertial regime $\left(\sigma_{\mathrm{s}} \leq 2 \bar{\Omega}_{\mathrm{s}}<N, v_{\mathrm{s}} \geq 1\right)$, waves (and the adiabatic wave operator) become hyperbolic and trapped in an equatorial belt where $\theta \in\left[\theta_{\mathrm{c}}, \pi-\theta_{\mathrm{c}}\right], \theta_{\mathrm{c}}$ being the critical colatitude

$\theta_{\mathrm{c}}=\cos ^{-1}\left(\frac{\sigma_{\mathrm{s}}}{2 \bar{\Omega}_{\mathrm{s}}}\right)$

where $\mathcal{D}=0$ and where the adiabatic wave velocity field (and operator) is singular. There, the traditional approximation cannot be applied (see Sect. 3.1 and references therein) and the regularization of the adiabatic wave velocity field is allowed by thermal and viscous diffusion that lead to shear layers, the attractors, where strong dissipation occurs that may induce transport and mixing (Dintrans et al. 1999; Dintrans \& Rieutord 2000). The description of this regime is out of the scope of this paper and should be examined in the near future.

In the case of a general strong differential rotation $(\Omega(r, x))$, the traditional approximation can be applied as long as $2 \Omega \ll N$ and $\sigma \ll N$ in spherical shell(s) where

$\mathcal{D}>0$ everywhere $(\forall r$ and $\forall x \in[-1,1])$

that corresponds to the regular elliptic gravito-inertial waves.

In the other spherical shell(s), where both $\mathcal{D} \leq 0$ and $\mathcal{D}>0$, critical surfaces appear, on which $\mathcal{D}=0$. Then, the traditional approximation fails to reproduce the wave behaviour since the adiabatic wave operator (and velocity field) becomes singular and thus it should be abandoned (Friedlander 1987), as in the sub-inertial regime in the weak differential rotation case.

To illustrate this, we consider the radiation zone of a solartype star. Its external border with the convective envelope, where a tachocline layer is assumed, is located at the radius $r=R_{\mathrm{T}}$ (in the Sun, $R_{\mathrm{T}} \approx 0.71 R_{\odot}$; see for example Schatzman et al. 2000). 


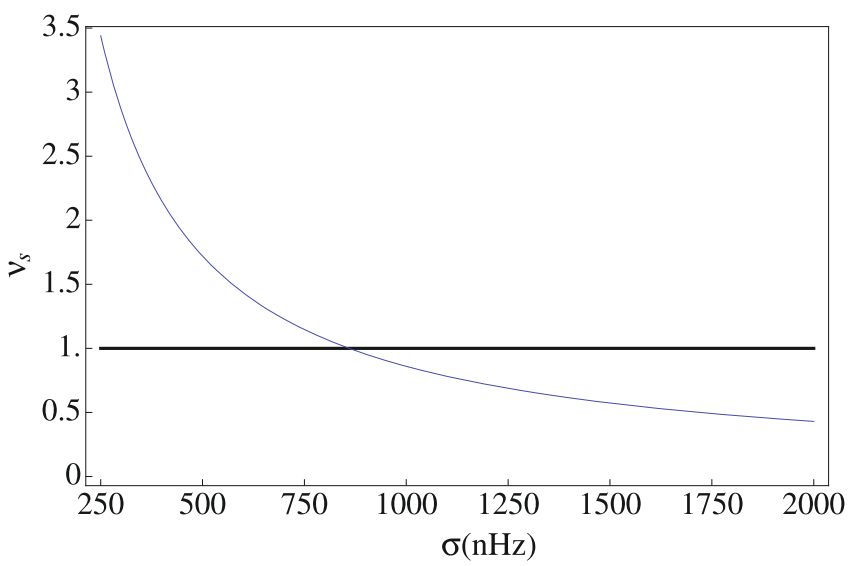

Fig. 2. $v_{\mathrm{s}}(\sigma)=2 \bar{\Omega}_{\mathrm{s}} / \sigma$ in the frequency range relevant for the calculation of angular momentum transport taking $\bar{\Omega}_{\mathrm{s}} / 2 \pi=430 \mathrm{nHz}$ for axisymmetric waves $(m=0)$. The traditional approximation is allowed when $v_{\mathrm{s}}<1$ and forbidden otherwise $\left(v_{\mathrm{s}} \geq 1\right)$.
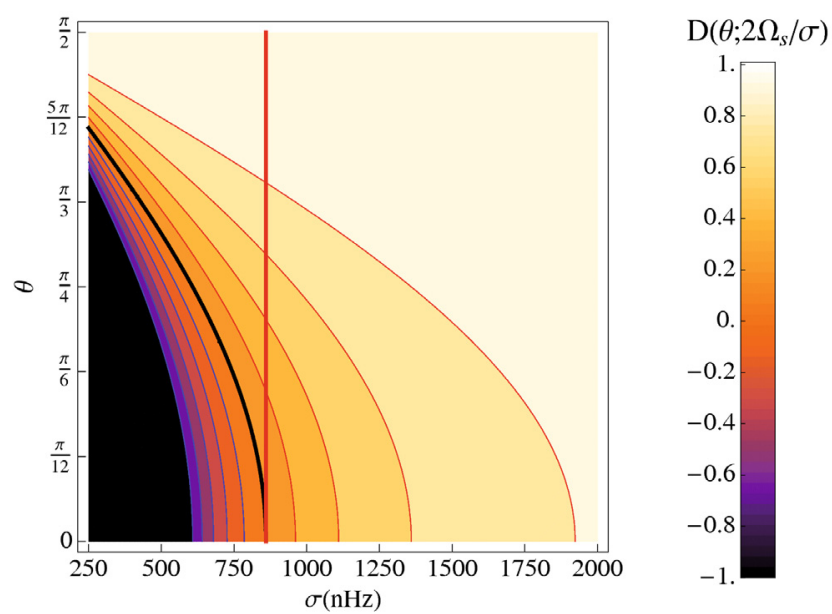

Fig. 3. $\mathcal{D}\left(\theta ; v_{\mathrm{s}}\right)$ as a function of $\theta$ and $\sigma$ for axisymmetric waves $(m=0)$. The critical surface $\mathcal{D}\left(\theta ; v_{\mathrm{s}}\right)=0$ (cf. Eq. (93)) is given by the thick black line and the iso- $\mathcal{D}$ lines such that $\mathcal{D}\left(\theta ; v_{\mathrm{s}}\right)>0$ and $\mathcal{D}\left(\theta ; v_{\mathrm{s}}\right)<0$ are respectively given by the red and the blue lines. The traditional approximation applies in spherical shell(s) such that $\mathcal{D}>0$ everywhere $(\forall r$ and $\forall \theta \in[0, \pi])$; there waves are regular at all latitudes. In other spherical shell(s), where both $\mathcal{D}>0$ and $\mathcal{D} \leq 0$, the traditional approximation does not apply due to the singularity where $\mathcal{D}=0$. Therefore, for $\bar{\Omega}_{\mathrm{s}}$, the traditional approximation applies in the domain in the $(\sigma, \theta)$ plane to the right of the vertical thick red line.

We consider three different angular velocity profiles.

First, we define a radial differential rotation, $\Omega_{1}(r)$, which has a smooth gradient troughout the radiative core:

$\Omega_{1}(r)=\bar{\Omega}_{\mathrm{S}}\left[1+\operatorname{sinc}\left(\pi \frac{r}{R_{\mathrm{T}}}\right)\right]$,

where $\operatorname{sinc}(X)=\sin X / X$ and $\bar{\Omega}_{\mathrm{s}}$ is solid-body rotation which is taken as the reference. Following Mathis et al. (2008), we choose $\bar{\Omega}_{\mathrm{s}} / 2 \pi=430 \mathrm{nHz}$.

Next, we consider a second radial differential rotation

$\Omega_{2}(r)=\bar{\Omega}_{\mathrm{s}}\left[2-A_{\mathrm{c}} \operatorname{Erf}\left(\frac{r-r_{\mathrm{c}}}{l_{\mathrm{c}}}\right)\right]$,

which has a strong gradient located in the core of the radiation zone $\left(r \in\left[0, R_{\mathrm{T}} / 3\right]\right)$ and a central rotation $\Omega_{2}(0)=3 \bar{\Omega}_{\mathrm{S}}$ (to obtain this profile we put $A_{\mathrm{c}}=1, r_{\mathrm{c}}=0.15 R_{\mathrm{T}}$ and $\left.l_{\mathrm{c}}=0.075 R_{\mathrm{T}}\right)$ as could be the case inside the Sun (Turck-Chièze et al. 2004;

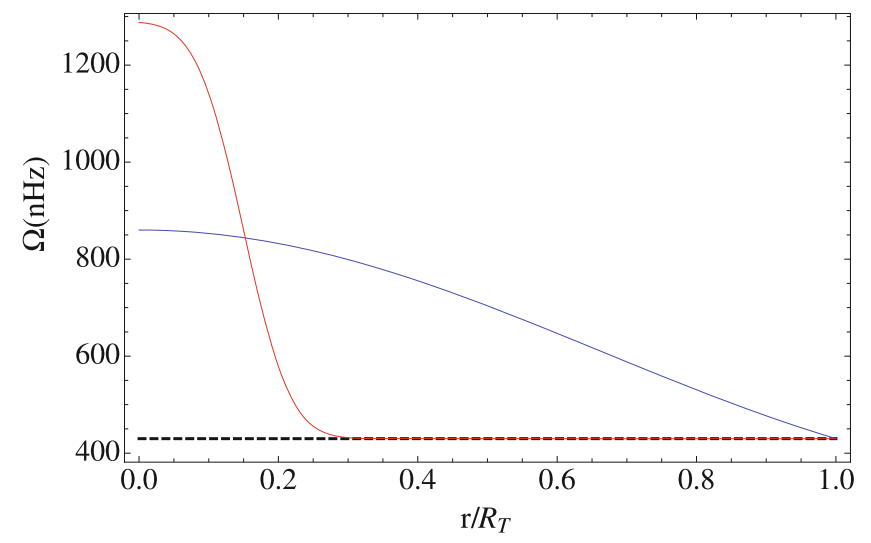

Fig. 4. Rotation frequencies $\Omega_{1}(r) / 2 \pi$ (blue line) and $\Omega_{2}(r) / 2 \pi$ (red line). The reference solid body rotation, $\bar{\Omega}_{\mathrm{s}} / 2 \pi$, is given by the thick dashed black line.

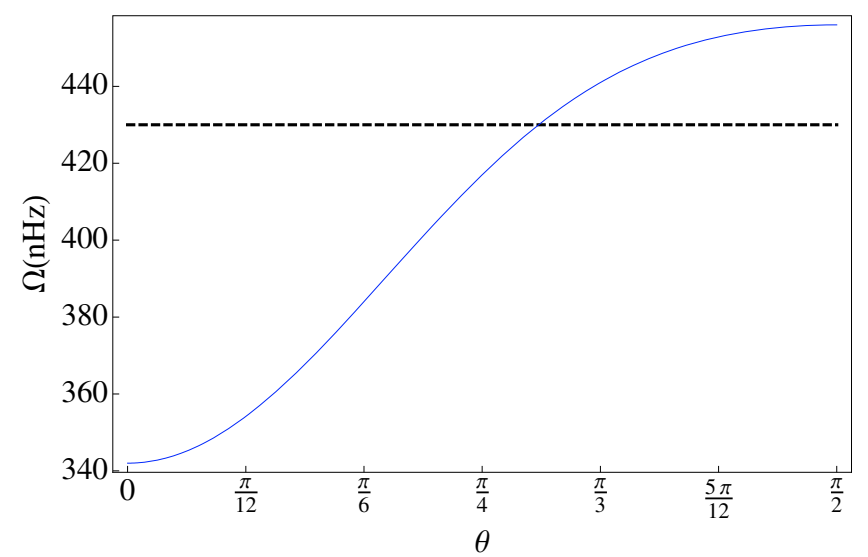

Fig. 5. Rotation frequency $\Omega_{3}(\theta) / 2 \pi$ (blue line). The reference solid body rotation, $\bar{\Omega}_{\mathrm{s}} / 2 \pi$, is given by the thick dashed black line.

Garcia et al. 2007; Mathur et al. 2008). $\operatorname{Erf}(X)$ is the classical error function (cf. Abramowitz \& Stegun 1972).

Finally, we study a third differential rotation that depends only on the colatitude $(\theta), \Omega_{3}(\theta)$, to illustrate the effect of the latitudinal gradient of the rotation frequency. We choose here the horizontal differential rotation obtained through helioseismic inversions at the bottom of the solar convective envelope (Thompson et al. 2003):

$\frac{\Omega_{3}(\theta)}{2 \pi}=A+B \cos ^{2} \theta+C \cos ^{4} \theta$,

where $A=456 \mathrm{nHz}, B=-42 \mathrm{nHz}$ and $C=-72 \mathrm{nHz}$.

First, $\widehat{v}$ is considered. In the case of radial differential rotation, $\Omega_{i}(r) \quad(i=\{1,2\})$, its variation is directly given by the rotation frequency profiles modulated by $1 / \widehat{\sigma}$ (cf. Fig. 6 where we focus on axisymmetric waves (i.e. $m=0$ ) that filters out the Doppler shift and thus allows us to isolate the effects of the differential rotation itself). Then, the surface $\widehat{v}=1$, which corresponds to $v_{\mathrm{s}}=1$ in the weak differential rotation case, is given by $\widehat{\sigma}=2 \Omega_{i}(r)$.

In the case of the latitudinal rotation, $\Omega_{3}(\theta)$, we obtain the same behaviour, the surface $\widehat{v}=1$ being given by $\widehat{\sigma}=2 \Omega_{3}(\theta)$ (cf. Fig. 7 for $m=0$ ).

In the case of a strong differential rotation, the traditional approximation can be applied in spherical shell(s) where (cf. Eq. (94))

$$
\begin{aligned}
1-\widehat{v}^{2} \cos ^{2} \theta-\widehat{v} \frac{\partial_{\theta} \Omega}{\widehat{\sigma}} & \cos \theta \sin \theta>0 \\
& \text { everywhere }(\forall r \text { and } \forall \theta \in[0, \pi]) .
\end{aligned}
$$



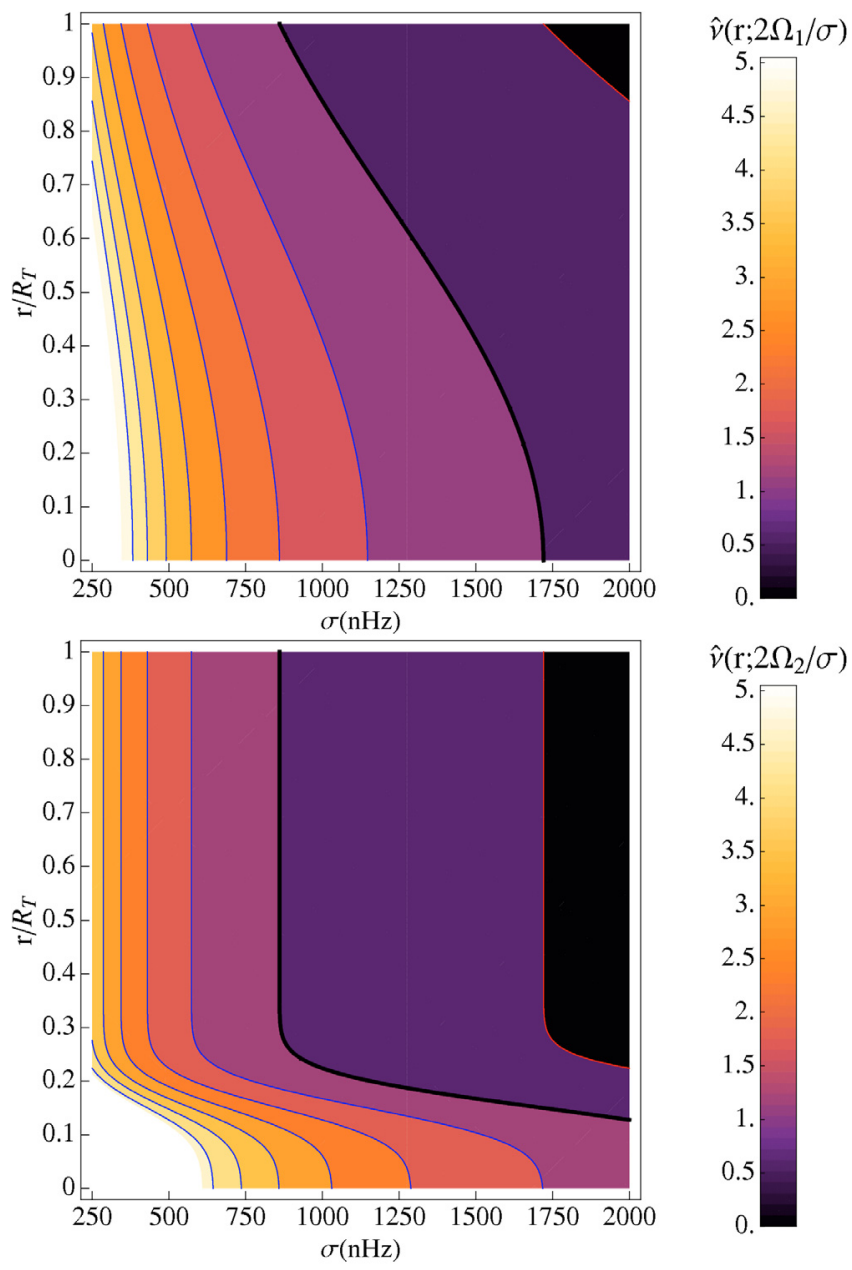

$\hat{v}\left(\mathrm{r} ; 2 \Omega_{2} / \sigma\right)$

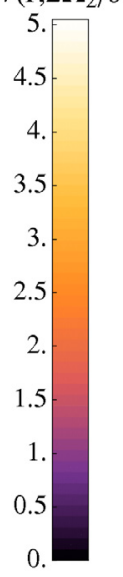

Fig. 6. Top: $\widehat{v}\left(r ; 2 \Omega_{1} / \sigma\right)$ as a function of $r$ and $\sigma$ for axisymmetric waves $(m=0)$. The surface $\widehat{v}\left(r ; 2 \Omega_{1} / \sigma\right)=1$ is given by the thick black line and the iso- $\widehat{v}$ lines such that $\widehat{v}\left(r ; 2 \Omega_{1} / \sigma\right)>1$ and $\widehat{v}\left(r ; 2 \Omega_{1} / \sigma\right)<1$ are respectively given by the blue and the red lines. Bottom: same for $\widehat{v}\left(r ; 2 \Omega_{2} / \sigma\right)$.

For a radial differential rotation, this corresponds to spherical shell(s) where

$$
4[\Omega(r)]^{2} \cos ^{2} \theta<\widehat{\sigma}^{2} \ll N^{2} \text { everywhere }(\forall r \text { and } \forall \theta \in[0, \pi])
$$

that leads to $2 \Omega(r)<\widehat{\sigma} \ll N$.

The cases of $\Omega_{1}$ and $\Omega_{2}$ are illustrated in Fig. 8 for $m=0$. In each of them, a forbidden spherical shell appears, where both $\mathcal{D} \leq 0$ and $\mathcal{D}>0$ (the adiabatic waves velocity field is singular where $\mathcal{D}=0$ ), that corresponds to an higher rotation frequency. Its spatial location and radius depend on the $\Omega_{i}(r)$ profile and it becomes smaller as the frequency increases.

Finally, in the case of a latitudinal differential rotation, such as $\Omega_{3}$, the allowed domain, in which the traditional approximation can be applied, is modified both by the rotation frequency profile and its latitudinal gradient. This is shown in Fig. 9 for $m=0$, which has to be compared with the weak differential rotation case studied in Fig. 3.

\section{Wave-induced transport of angular momentum}

From now on, we study the wave-induced transport of energy and of angular momentum in spherical shell(s) where the traditional approximation can be applied. In other words, we focus on the transport associated with the regular elliptic gravito-inertial
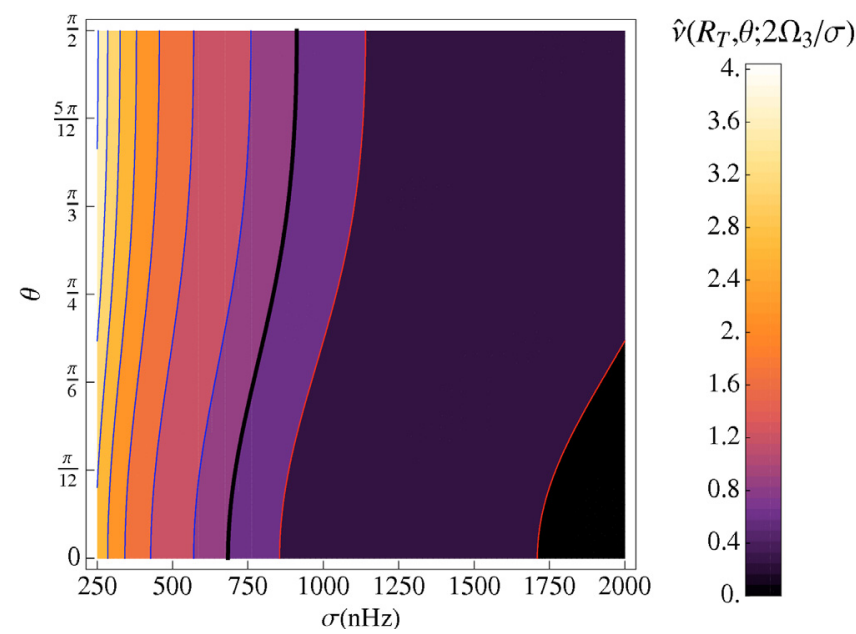

Fig. 7. $\widehat{v}\left(R_{\mathrm{T}}, \theta ; 2 \Omega_{3} / \sigma\right)$ as a function of $\theta$ and $\sigma$ for axisymmetric waves $(m=0)$. The surface $\widehat{v}\left(R_{\mathrm{T}}, \theta ; 2 \Omega_{3} / \sigma\right)=1$ is given by the thick black line and the iso- $\widehat{v}$ lines with $\widehat{v}\left(R_{\mathrm{T}}, \theta ; 2 \Omega_{3} / \sigma\right)>1$ and $\widehat{v}\left(R_{\mathrm{T}}, \theta ; 2 \Omega_{3} / \sigma\right)<$ 1 are respectively given by the blue and the red lines.

waves, the hyperbolic regime being beyond the scope of this paper.

Therefore, since we are now working in allowed spherical shell(s), all the classical averages over longitudes $(\varphi)$ and colatitudes $(\theta)$ can be defined.

\subsection{Fluxes transported by a monochromatic wave}

The goal of this paper is to study the influence of a general differential rotation on low-frequency waves and their feed-back on the transport of angular momentum. The first step in this part of the work is now to derive the fluxes of energy and of angular momentum carried by a monochromatic wave.

\subsubsection{Fluxes of energy}

In the general bidimensional case which is studied here, the flux of energy in the direction of the $k$ th coordinate is given by the acoustic flux (see Lighthill 1978; Press 1981; Unno et al. 1989) ${ }^{5}$

$\mathcal{F}_{k ; j, m}^{\mathrm{E}}=\left\langle P_{j, m}^{\prime} u_{k ; j, m}\right\rangle_{\varphi}$,

where $P_{j, m}^{\prime}$ and the $u_{k ; j, m}$ components (where $k=\{r, \theta, \varphi\}$ ) were obtained in the previous section and $\langle\ldots\rangle_{\varphi}=\frac{1}{2 \pi} \int_{0}^{2 \pi} \ldots \mathrm{d} \varphi$.

${ }^{5}$ Following the derivation given in Unno et al. (1989), we get the wave-energy equation first derived by Ando (1985):

$$
\left(\partial_{t}+\Omega \partial_{\varphi}\right) E+\boldsymbol{\nabla} \cdot \boldsymbol{F}^{\mathrm{E}}=-\widetilde{\phi} \partial_{t} \widetilde{\rho}-\bar{\rho} r \sin \theta(\boldsymbol{u} \cdot \boldsymbol{\nabla} \Omega) u_{\varphi},
$$

where the energy (E) and the wave-energy flux $\left(\boldsymbol{F}^{\mathrm{E}}\right)$ are given by:

$$
E=\frac{1}{2} \bar{\rho}[\underbrace{\boldsymbol{u}^{2}}_{1}+\underbrace{\left(\frac{\widetilde{P}}{\bar{\rho} c_{\mathrm{s}}}\right)^{2}+\left(\frac{\boldsymbol{g}}{N}\right)^{2}\left(\frac{\widetilde{P}}{\Gamma_{1} \bar{P}}-\frac{\widetilde{\rho}}{\bar{\rho}}\right)^{2}}_{2}]
$$

and

$$
\boldsymbol{F}^{\mathrm{E}}=\widetilde{P} \boldsymbol{u}+\bar{\rho} \boldsymbol{u} \widetilde{\phi}
$$

$c_{\mathrm{s}}^{2}=\Gamma_{1} \frac{\bar{P}}{\bar{\rho}}$ being the sound-speed. Terms 1 and 2 correspond to the kinetic and the potential energies.

When the Cowling's approximation is assumed, the wave-energy flux thus reduces to the acoustic flux: $\boldsymbol{F}^{\mathrm{E}}=\widetilde{P} \boldsymbol{u}$. 

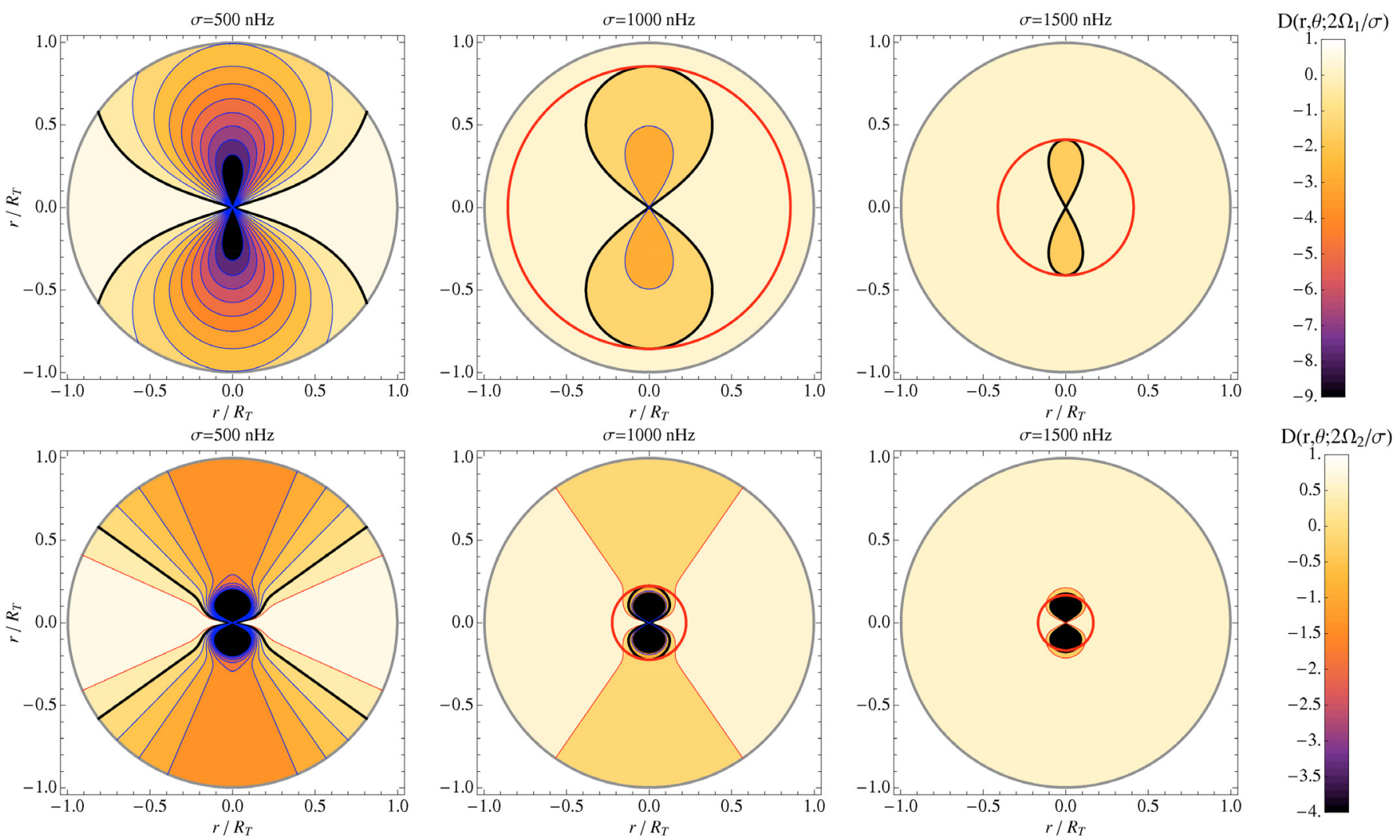

Fig. 8. Top: $\mathcal{D}\left(r, \theta ; 2 \Omega_{1} / \sigma\right)$ as a function of $r$ and $\theta$ for $\sigma=500,1000,1500 \mathrm{nHz}$ for axisymmetric waves $(m=0)$. The critical surface $\mathcal{D}\left(r, \theta ; 2 \Omega_{1} / \sigma\right)=0$ is given by the thick black line and the iso- $\mathcal{D}$ lines for $\mathcal{D}\left(r, \theta ; 2 \Omega_{1} / \sigma\right)>0$ and $\mathcal{D}\left(r, \theta ; 2 \Omega_{1} / \sigma\right)<0$ are given by the red and the blue lines. The traditional approximation applies in spherical shell(s) such that $\mathcal{D}>0$ everywhere $(\forall r$ and $\forall \theta \in[0, \pi])$; there, waves are regular at all latitudes. In other spherical shell(s), where both $\mathcal{D}>0$ and $\mathcal{D} \leq 0$, the traditional approximation does not apply due to the singularity where $\mathcal{D}=0$. Therefore, for $\Omega_{1}$, the traditional approximation does not apply for $\sigma / 2 \pi=500 \mathrm{nHz}$ while it applies for $\sigma / 2 \pi=1000 \& 1500 \mathrm{nHz}$ in the external spherical shell with the inner border given by the thick red circle. Bottom: same for $\mathcal{D}\left(r, \theta ; 2 \Omega_{2} / \sigma\right)$.

\section{Radial flux of energy}

The flux of energy transported in the radial direction is thus given by

$\mathcal{F}_{V ; j, m}^{\mathrm{E}}(r, \theta)=\left\langle P_{j, m}^{\prime} u_{r ; j, m}\right\rangle_{\varphi}$.

Using the expression derived in Eqs. (72) and (75), we thus obtain:

$\mathcal{F}_{V ; j, m}^{\mathrm{E}}=-\frac{1}{2} \bar{\rho} \frac{\widehat{\sigma}}{N} \frac{\lambda_{j, m}^{1 / 2}}{r} w_{j, m}^{2} \exp \left[-\tau_{j, m}\right]$.

\section{Horizontal flux of energy}

In the same way, the flux of energy transported in the horizontal direction is given by the sum of fluxes in the latitudinal and azimuthal directions:

$\mathcal{F}_{H ; j, m}^{\mathrm{E}}(r, \theta)=\mathcal{F}_{\theta ; j, m}^{\mathrm{E}}(r, \theta)+\mathcal{F}_{\varphi ; j, m}^{\mathrm{E}}(r, \theta)$

where

$\mathcal{F}_{\theta ; j, m}^{\mathrm{E}}=\left\langle P_{j, m}^{\prime} u_{\theta ; j, m}\right\rangle_{\varphi} \quad$ and $\quad \mathcal{F}_{\varphi ; j, m}^{\mathrm{E}}=\left\langle P_{j, m}^{\prime} u_{\varphi ; j, m}\right\rangle_{\varphi}$.

Using Eqs. $(72,77)$, we get:

$\mathcal{F}_{\theta ; j, m}^{\mathrm{E}}=0$

due to the quadrature between $P_{j, m}^{\prime}$ and $u_{\theta ; j, m}$ and finally:

$\mathcal{F}_{H ; j, m}^{\mathrm{E}}=\mathcal{F}_{\varphi ; j, m}^{\mathrm{E}}=-\frac{1}{2} \bar{\rho} \frac{\widehat{\sigma}}{r} w_{j, m} \mathcal{G}_{j, m}^{\varphi} \exp \left[-\tau_{j, m}\right]$.

\subsubsection{Fluxes of angular momentum}

The equation for the transport of angular momentum is given by (see for example Brun \& Toomre 2002; or Mathis \& Zahn 2004):

$$
\begin{aligned}
\bar{\rho} \frac{\mathrm{d}}{\mathrm{d} t}\left(r^{2} \sin ^{2} \theta \Omega\right)+\nabla \cdot\left[\bar{\rho} r^{2} \sin ^{2} \theta \Omega \mathcal{U}_{M}(r, \theta)\right]= \\
\frac{\sin ^{2} \theta}{r^{2}} \partial_{r}\left(\bar{\rho} v_{V} r^{4} \partial_{r} \Omega\right)+\frac{1}{\sin \theta} \partial_{\theta}\left(\bar{\rho} v_{H} \sin ^{3} \theta \partial_{\theta} \Omega\right) \\
-\frac{1}{r^{2}} \partial_{r}\left[r^{2} \mathcal{F}_{V}^{\mathrm{AM}}(r, \theta)\right]-\frac{1}{r \sin \theta} \partial_{\theta}\left[\sin \theta \mathcal{F}_{H}^{\mathrm{AM}}(r, \theta)\right] .
\end{aligned}
$$

Since this work is dedicated to the secular rotational transport during the evolution of the star, the Lagrangian time derivative $\mathrm{d} / \mathrm{d} t=\partial_{t}+\dot{r} \partial_{r}$ is kept, meaning that the radial coordinate $r$ is the mean radius of the layer (the isobar) enclosing the mass $M_{r}$ with $\mathrm{d} M_{r}=4 \pi \bar{\rho} r^{2} \mathrm{~d} r \cdot \dot{r} \widehat{\boldsymbol{e}}_{r}$ is the radial velocity field that corresponds to the contractions and dilatations of the star during its evolution. The second term on the left-hand side corresponds to the flux of angular momentum, which is advected by the meridional circulation, $\boldsymbol{U}_{M}$. Then, as in Zahn (1992), we assume that the effect of the turbulent stresses on the large-scale flows are adequately described by an anisotropic eddy-viscosity, whose components are respectively $v_{V}$ and $v_{H}$ in the radial and the horizontal directions. In stellar radiation zones, they act to reduce their cause, namely the radial and horizontal gradients of angular velocity. Finally, $\mathcal{F}_{V}^{\mathrm{AM}}$ and $\mathcal{F}_{H}^{\mathrm{AM}}$ are respectively the radial and the horizontal components of the Lagrangian flux of angular momentum 

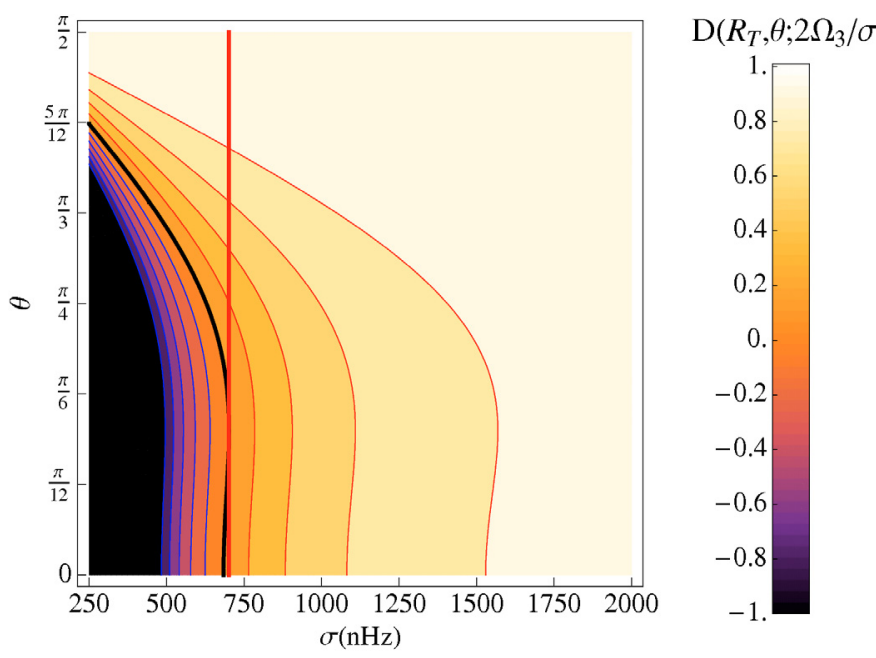

Fig. 9. $\mathcal{D}\left(R_{\mathrm{T}}, \theta ; 2 \Omega_{3} / \sigma\right)$ as a function of $\theta$ and $\sigma$ for axisymmetric waves $(m=0)$. The critical surface $\mathcal{D}\left(R_{\mathrm{T}}, \theta ; 2 \Omega_{3} / \sigma\right)=0$ is given by the thick black line and the iso- $\mathcal{D}$ lines for $\mathcal{D}\left(R_{\mathrm{T}}, \theta ; 2 \Omega_{3} / \sigma\right)>0$ and $\mathcal{D}\left(R_{\mathrm{T}}, \theta ; 2 \Omega_{3} / \sigma\right)<0$ are respectively given by the red and the blue lines. The traditional approximation applies in spherical shell(s) such that $\mathcal{D}>0$ everywhere $(\forall r$ and $\forall \theta \in[0, \pi])$; there, waves are regular at all latitudes. In other spherical shell(s), where both $\mathcal{D}>0$ and $\mathcal{D} \leq 0$, the traditional approximation does not apply due to the singularity where $\mathcal{D}=0$. Therefore, for $\Omega_{3}$, the traditional approximation applies in the domain in the $(\sigma, \theta)$ plane to the right of the vertical thick red line.

transported by the Reynolds stresses of the IGWs ${ }^{6}$ :

$$
\begin{aligned}
\mathcal{F}_{V}^{\mathrm{AM}} & =\langle\bar{\rho} r \sin \theta u_{r} u_{\varphi}+\underbrace{\bar{\rho} r \sin \theta 2 \Omega \cos \theta u_{r} \xi_{\theta}}_{L}\rangle_{\varphi}, \\
\mathcal{F}_{H}^{\mathrm{AM}} & =\left\langle\bar{\rho} r \sin \theta u_{\theta} u_{\varphi}\right\rangle_{\varphi} .
\end{aligned}
$$

\section{Radial component of the flux of angular momentum}

The radial component of the monochromatic flux of angular momentum is then given by:

$$
\begin{aligned}
\mathcal{F}_{V ; j, m}^{\mathrm{AM}}(r, \theta)= & \left\langle\bar{\rho} r \sin \theta u_{r ; j, m} u_{\varphi ; j, m}\right. \\
& \left.+\bar{\rho} r \sin \theta 2 \Omega \cos \theta u_{r ; j, m} \xi_{\theta ; j, m}\right\rangle_{\varphi}
\end{aligned}
$$

that becomes, once again using Eqs. $(75,77,79)$ :

$$
\begin{aligned}
\mathcal{F}_{V ; j, m}^{\mathrm{AM}}= & \frac{1}{2} \bar{\rho} r \sin \theta \frac{1}{N} \frac{\lambda_{j, m}^{1 / 2}(r ; \widehat{v})}{r^{2}} \\
& \times \widehat{\sigma}^{2} w_{j, m}\left(\mathcal{G}_{j, m}^{\varphi}-\widehat{v} \cos \theta \mathcal{G}_{j, m}^{\theta}\right) \exp \left[-\tau_{j, m}\right] .
\end{aligned}
$$

Following Zahn et al. (1997), the mean vertical flux of angular momentum on an isobar is defined:

$\overline{\mathcal{F}_{V ; j, m}^{\mathrm{AM}}}(r)=\frac{1}{\int_{0}^{\pi} \sin ^{3} \theta \mathrm{d} \theta}\left\langle\mathcal{F}_{V ; j, m}^{\mathrm{AM}}\right\rangle_{\theta}$,

where $\langle\ldots\rangle_{\theta}=\int_{0}^{\pi} \ldots \sin \theta \mathrm{d} \theta$. We obtain:

$$
\begin{aligned}
& \overline{\mathcal{F}_{V ; j, m}^{\mathrm{AM}}}=\frac{3}{8} \bar{\rho} r \frac{1}{N} \frac{\lambda_{j, m}^{1 / 2}(r ; \widehat{v})}{r^{2}} \\
& \quad \times\left\langle\sin \theta \widehat{\sigma}^{2} w_{j, m}\left(\mathcal{G}_{j, m}^{\varphi}-\widehat{v} \cos \theta \mathcal{G}_{j, m}^{\theta}\right) \exp \left[-\tau_{j, m}\right]\right\rangle_{\theta} .
\end{aligned}
$$

${ }^{6}$ The additional term $L$ in $\mathcal{F}_{V}^{\mathrm{AM}}$ has been discussed by Bretherton (1969) and added by Pantillon et al. (2007). It corresponds to the Lagrangian flux of angular momentum through a level surface in a rotating system.

\section{Horizontal component of the flux of angular momentum}

In the same way, we derive the latitudinal component of the monochromatic flux of angular momentum:

$\mathcal{F}_{H ; j, m}^{\mathrm{AM}}(r, \theta)=\left\langle\bar{\rho} r \sin \theta u_{\theta ; j, m} u_{\varphi ; j, m}\right\rangle_{\varphi}=0$,

due to the quadrature between $u_{\theta ; j, m}$ and $u_{\varphi ; j, m}$.

\subsubsection{Action (luminosity) of angular momentum}

We can define the monochromatic action of angular momentum (that is called luminosity of angular momentum in stellar physics)

$\mathcal{L}_{V ; j, m}^{\mathrm{AM}}(r, \theta)=r^{2} \mathcal{F}_{V ; j, m}^{\mathrm{AM}}$.

In the adiabatic case, where the radiative damping is not taken into account, this action of angular momentum (as well as the action of energy, namely $r^{2} \mathcal{F}_{V ; j, m}^{\mathrm{E}}$ ) is conserved as demonstrated by Hayes (1970) and Goldreich \& Nicholson (1989a). In the adiabatic case, we thus obtain:

$\mathcal{L}_{V ; j, m}^{\mathrm{AM}}(r, \theta)=\mathcal{L}_{V ; j, m}^{\mathrm{AM}}\left(r_{\mathrm{c}}, \theta\right)$,

where $r_{\mathrm{c}}$ is the radius of the position of the border between the radiative region and the convective one that excites the waves. In the quasi-adiabatic case, this becomes:

$\mathcal{L}_{V ; j, m}^{\mathrm{AM}}(r, \theta)=\mathcal{L}_{V ; j, m}^{\mathrm{AM}}\left(r_{\mathrm{c}}, \theta\right) \exp \left[-\tau_{j, m}\right]$

that gives

$$
\begin{aligned}
& \mathcal{L}_{V ; j, m}^{\mathrm{AM}}=\frac{1}{2} \bar{\rho}_{\mathrm{c}} r_{\mathrm{c}}^{3} \frac{1}{N_{\mathrm{c}}} \frac{\lambda_{j, m}^{1 / 2}\left(r_{\mathrm{c}} ; \widehat{v}_{\mathrm{c}}\right)}{r_{\mathrm{c}}^{2}} \\
& \quad \times \sin \theta\left[\widehat{\sigma}^{2} w_{j, m}\left(\mathcal{G}_{j, m}^{\varphi}-\widehat{v} \cos \theta \mathcal{G}_{j, m}^{\theta}\right)\right]_{r=r_{\mathrm{c}}} \exp \left[-\tau_{j, m}\right]
\end{aligned}
$$

where

$$
\begin{aligned}
{\left[\widehat{\sigma}^{2} w_{j, m}\left(\mathcal{G}_{j, m}^{\varphi}-\widehat{v} \cos \theta \mathcal{G}_{j, m}^{\theta}\right)\right]_{r=r_{\mathrm{c}}}=} \\
\widehat{\sigma}_{\mathrm{CZ}}^{2}\left(r_{\mathrm{c}}, \theta\right) w_{j, m}\left(r_{\mathrm{c}}, \theta ; \widehat{v}_{\mathrm{c}}\right) \\
\times\left[\mathcal{G}_{j, m}^{\varphi}\left(r_{\mathrm{c}}, \theta ; \widehat{v}_{\mathrm{c}}\right)-\widehat{v}_{\mathrm{c}}(\theta) \cos \theta \mathcal{G}_{j, m}^{\theta}\left(r_{\mathrm{c}}, \theta ; \widehat{v}_{\mathrm{c}}\right)\right] .
\end{aligned}
$$

We have defined the local spin parameter at $r=r_{\mathrm{c}}$

$\widehat{v}_{\mathrm{c}}(\theta)=\widehat{v}\left(r_{\mathrm{c}}, \theta\right)=\frac{2 \Omega\left(r_{\mathrm{c}}, \theta\right)}{\widehat{\sigma}\left(r_{\mathrm{c}}, \theta\right)}=\frac{2 \Omega_{\mathrm{CZ}}\left(r_{\mathrm{c}}, \theta\right)}{\widehat{\sigma}_{\mathrm{CZ}}\left(r_{\mathrm{c}}, \theta\right)}$

where

$\widehat{\sigma}_{\mathrm{CZ}}\left(r_{\mathrm{c}}, \theta\right)=\sigma+m \Omega_{\mathrm{CZ}}\left(r_{\mathrm{c}}, \theta\right)$,

$\Omega_{\mathrm{CZ}}(r, \theta)$ being the angular velocity of the convection zone.

As in Eqs. $(111,112)$, the mean action of angular momentum on an isobar is derived:

$\overline{\mathcal{L}_{V ; j, m}^{\mathrm{AM}}}(r)=r^{2} \overline{\mathcal{F}_{V ; j, m}^{\mathrm{AM}}}$.

We thus obtain:

$$
\begin{aligned}
& \overline{\mathcal{L}_{V ; j, m}^{\mathrm{AM}}}=\frac{3}{8} \bar{\rho}_{\mathrm{c}} r_{\mathrm{c}}^{3} \frac{1}{N_{\mathrm{c}}} \frac{\lambda_{j, m}^{1 / 2}\left(r_{\mathrm{c}} ; \widehat{\nu}_{\mathrm{c}}\right)}{r_{\mathrm{c}}^{2}} \\
& \quad \times\left\langle\sin \theta\left[\widehat{\sigma}^{2} w_{j, m}\left(\mathcal{G}_{j, m}^{\varphi}-\widehat{v} \cos \theta \mathcal{G}_{j, m}^{\theta}\right)\right]_{r=r_{\mathrm{c}}} \exp \left[-\tau_{j, m}\right]\right\rangle_{\theta} .
\end{aligned}
$$

To derive the total angular momentum flux transported by IGWs, the match between the turbulent convection and the waves now has to be examined very carefully to obtain a correct treatment of their excitation. 


\subsection{Waves excitation by convection and total action of angular momentum}

\subsubsection{Energy flux transfer}

The first method to treat the wave excitation problem is to relate the flux of angular momentum to the wave-energy flux. Following the procedure used for the non-rotating and the weak differential rotation cases (cf. Zahn et al. 1997; Pantillon et al. 2007), we define $\widehat{m}$ such that:

$\mathcal{F}_{V ; j, m}^{\mathrm{AM}}=-\frac{\widehat{m}(r, x ; \widehat{v})}{\widehat{\sigma}} \mathcal{F}_{V ; j, m}^{\mathrm{E}}$.

Using the respective expressions of $\mathcal{F}_{V ; j, m}^{\mathrm{E}}$ and $\mathcal{F}_{V ; j, m}^{\mathrm{AM}}$ given in Eqs. (101) and (109), we get

$\widehat{m}(r, x ; \widehat{v})=\frac{\sin \theta \widehat{\sigma}^{2} w_{j, m}\left[\mathcal{G}_{j, m}^{\varphi}-\widehat{v} \cos \theta \mathcal{G}_{j, m}^{\theta}\right]}{w_{j, m}^{2}}$.

This can be understood as the efficiency transmission factor that gives us, for each frequency and each latitude, the energy transfer from the convective movements to the waves. It also allows us to quantify the bias in the excitation between prograde and retrograde waves.

In the non-rotating case $(\Omega=0)$, we get $\widehat{m}=m$ and therefore

$\mathcal{F}_{V ; l, m}^{\mathrm{AM}}=-\frac{m}{\sigma} \mathcal{F}_{V ; l, m}^{\mathrm{E}}=-2 \frac{m}{\sigma} \mathcal{F}_{V ; l, m}^{\mathrm{K}}$,

where $\mathcal{F}_{V ; l, m}^{\mathrm{K}}$ is the kinetic energy flux (in the case of lowfrequency gravity waves, the energy equipartition is obtained so that $\mathcal{F}_{V ; l, m}^{\mathrm{E}}=2 \mathcal{F}_{V ; l, m}^{\mathrm{K}}$; on the other hand $j$ reduces to the classical orbital number of spherical harmonics, $l$ ).

Using Eq. (115), we thus have:

$\mathcal{L}_{V ; j, m}^{\mathrm{AM}}(r, x ; \widehat{v})=\mathcal{L}_{V ; j, m}^{\mathrm{AM}}\left(r_{\mathrm{c}}, x ; \widehat{v}_{\mathrm{c}}\right) \exp \left[-\tau_{j, m}\right]$

where

$\mathcal{L}_{V ; j, m}^{\mathrm{AM}}\left(r_{\mathrm{c}}, x ; \widehat{v}_{\mathrm{c}}\right)=-r_{\mathrm{c}}^{2} \frac{\widehat{m}\left(r_{\mathrm{c}}, x ; \widehat{v}_{\mathrm{c}}\right)}{\widehat{\sigma}_{\mathrm{CZ}}} \mathcal{F}_{V ; j, m}^{\mathrm{E}}\left(r_{\mathrm{c}}, x ; \widehat{v}_{\mathrm{c}}\right)$.

Taking all the spectrum of excited waves, $\mathcal{L}_{V}^{\mathrm{AM}}$ and its associated average on an isobar are finally given by

$$
\begin{aligned}
& \mathcal{L}_{V}^{\mathrm{AM}}(r, x ; \widehat{v})= \\
& \quad-r_{\mathrm{c}}^{2} \int_{\sigma} \sum_{m, j}\left\{\frac{\widehat{m}\left(r_{\mathrm{c}}, x ; \widehat{v}_{\mathrm{c}}\right)}{\widehat{\sigma}_{\mathrm{CZ}}} \mathcal{F}_{V ; j, m}^{\mathrm{E}}\left(r_{\mathrm{c}}, x ; \widehat{v}_{\mathrm{c}}\right) \exp \left[-\tau_{j, m}\right]\right\} \mathrm{d} \sigma(
\end{aligned}
$$

and

$$
\begin{aligned}
& \overline{\mathcal{L}_{V}^{\mathrm{AM}}}(r)= \\
& -r_{\mathrm{c}}^{2} \int_{\sigma} \sum_{m, j}\left\{\left\langle\frac{\widehat{m}\left(r_{\mathrm{c}}, x ; \widehat{v}_{\mathrm{c}}\right)}{\widehat{\sigma}_{\mathrm{CZ}}} \mathcal{F}_{V ; j, m}^{\mathrm{E}}\left(r_{\mathrm{c}}, x ; \widehat{v}_{\mathrm{c}}\right) \exp \left[-\tau_{j, m}\right]\right\rangle_{\theta}\right\} \mathrm{d} \sigma .
\end{aligned}
$$

The transported flux of angular momentum now being derived, it is necessary to look for a robust prescription for the excited wave energy spectrum at $r=r_{\mathrm{c}}$. This will give the excited frequencies, that are crucial for the waves damping (cf. Eq. (68)) which rules the transport of angular momentum, and the associated energy flux $\mathcal{F}_{V ; j, m}^{\mathrm{E}}\left(r_{\mathrm{c}}, x ;{\widehat{v_{\mathrm{c}}}}_{\mathrm{c}}\right)$. This will be discussed in Sect. 4.2.3.

\subsubsection{Amplitude of each monochromatic wave}

The second method to approach the problem of wave excitation is to work on the amplitude of each monochromatic wave.

We assume that the pressure field in the convection zone at $r=r_{\mathrm{c}}$ can be expanded formally in the following Fourier form:

$$
\begin{aligned}
P_{\mathrm{CZ}}\left(r_{\mathrm{c}}, \theta, \varphi, t\right)=\bar{\rho} \int_{\sigma} & \sum_{m}\left\{W_{\mathrm{CZ} ; m}\left(r_{\mathrm{c}}, \theta ; \sigma\right)\right. \\
& \times \exp [\mathrm{i}(m \varphi+\sigma t)]\} \mathrm{d} \sigma,
\end{aligned}
$$

where the $W_{\mathrm{CZ} ; m}$ are the Fourier coefficients in time of $P_{\mathrm{CZ}}$ for each $m$. Since, the $\left\{w_{j, m}\right\}_{j, m}$ form a complete orthogonal basis, $W_{\mathrm{CZ} ; m}$ can be projected on them:

$W_{\mathrm{CZ} ; m}\left(r_{\mathrm{c}}, \theta ; \sigma\right)=\sum_{j} a_{j, m}\left(r_{\mathrm{c}} ; \sigma\right) w_{j, m}\left(r_{\mathrm{c}}, \theta ; \widehat{v}_{\mathrm{c}}\right)$,

where the $a_{j, m}$ projection coefficients are given by:

$a_{j, m}\left(r_{\mathrm{c}} ; \sigma\right)=\frac{\left\langle W_{\mathrm{CZ} ; m}\left(r_{\mathrm{c}}, \theta ; \sigma\right) w_{j, m}\left(r_{\mathrm{c}}, \theta ; \widehat{v}_{\mathrm{c}}\right)\right\rangle_{\theta}}{\left\langle\left[w_{j, m}\left(r_{\mathrm{c}}, \theta ; \widehat{v}_{\mathrm{c}}\right)\right]^{2}\right\rangle_{\theta}}$

Assuming the continuity of the pressure between the turbulent movements in the convection zone and the waves inside the radiative region, summing over the spectrum of excited frequencies, the total action of angular momentum associated with waves is derived

$$
\begin{aligned}
& \mathcal{L}_{V}^{\mathrm{AM}}=\frac{1}{2} \bar{\rho}_{\mathrm{c}} r_{\mathrm{c}} \frac{1}{N_{\mathrm{c}}} \int_{\sigma} \sum_{m, j}\left\{\lambda_{j, m}^{1 / 2}\left(r_{\mathrm{c}} ; \widehat{v}_{\mathrm{c}}\right) a_{j, m}^{2}\left(r_{\mathrm{c}} ; \sigma\right)\right. \\
& \left.\quad \times \sin \theta\left[\widehat{\sigma}^{2} w_{j, m}\left(\mathcal{G}_{j, m}^{\varphi}-\widehat{v} \cos \theta \mathcal{G}_{j, m}^{\theta}\right)\right]_{r=r_{\mathrm{c}}} \exp \left[-\tau_{j, m}\right]\right\} \mathrm{d} \sigma
\end{aligned}
$$

with its associated average on an isobar

$$
\begin{aligned}
& \overline{\mathcal{L}_{V}^{\mathrm{AM}}}=\frac{3}{8} \bar{\rho}_{\mathrm{c}} r_{\mathrm{c}} \frac{1}{N_{\mathrm{c}}} \int_{\sigma}\left\{\sum_{m, j} \lambda_{j, m}^{1 / 2}\left(r_{\mathrm{c}} ; \widehat{v}_{\mathrm{c}}\right) a_{j, m}^{2}\left(r_{\mathrm{c}} ; \sigma\right)\right. \\
& \left.\quad \times\left\langle\sin \theta\left[\widehat{\sigma}^{2} w_{j, m}\left(\mathcal{G}_{j, m}^{\varphi}-\widehat{v} \cos \theta \mathcal{G}_{j, m}^{\theta}\right)\right]_{r=r_{\mathrm{c}}} \exp \left[-\tau_{j, m}\right]\right\rangle_{\theta}\right\} \mathrm{d} \sigma,
\end{aligned}
$$

where $N_{\mathrm{c}}=N\left(r_{\mathrm{c}}\right)$ is non-zero due to the convective penetration and the overshoot (see Zahn 1991). We are now looking for a robust prescription for $P_{\mathrm{CZ}}$. To achieve this aim, the different approaches reviewed in Sect. 4.2.3 are examined.

In every case, the pressure field at $r=r_{\mathrm{c}}$ can be expanded in spherical harmonics:

$$
\begin{aligned}
& P_{\mathrm{CZ}}\left(r_{\mathrm{c}}, \theta, \varphi, t\right)= \\
& \quad \bar{\rho} \int_{\sigma} \sum_{l, m}\left\{W_{\mathrm{CZ} ; l, m}\left(r_{\mathrm{c}} ; \sigma\right) \widetilde{P}_{l}^{m}(\cos \theta) \exp [\mathrm{i}(m \varphi+\sigma t)]\right\} \mathrm{d} \sigma,(134)
\end{aligned}
$$

where the normalized associated Legendre polynomials have been defined:

$\widetilde{P}_{l}^{m}(\cos \theta)=(-1)^{\frac{m+|m|}{2}}\left[\frac{2 l+1}{4 \pi} \frac{(l-|m|) !}{(l+|m|) !}\right]^{\frac{1}{2}} P_{l}^{m}(\cos \theta)$.

The $W_{\mathrm{CZ} ; l, m}$ are the Fourier coefficients in time of $P_{\mathrm{CZ}}$ for each spherical function. Then, the procedure that has allowed us to 
derive the general formal result given in Eq. (132) is applied. We thus project $P_{\mathrm{CZ}}$ on the $\left\{w_{j, m}\right\}_{j, m}$ basis:

$$
\begin{aligned}
P_{\mathrm{CZ}}\left(r_{\mathrm{c}}, \theta, \varphi, t\right)= & \bar{\rho} \int_{\sigma} \sum_{l, m} \sum_{j}\left\{a_{j, m}\left(r_{\mathrm{c}} ; \sigma\right) w_{j, m}\left(r_{\mathrm{c}}, \theta ; \widehat{v}_{\mathrm{c}}\right)\right. \\
& \times \exp [\mathrm{i}(m \varphi+\sigma t)]\} \mathrm{d} \sigma,
\end{aligned}
$$

where

$a_{j, m}\left(r_{\mathrm{c}} ; \sigma\right)=W_{\mathrm{CZ} ; l, m}\left(r_{\mathrm{c}} ; \sigma\right) \mathcal{P}_{l, m}^{j}\left(r_{\mathrm{c}} ; \widehat{v}_{\mathrm{c}}\right)$,

the projection of each spherical function on the $w_{j, m}$ being given by:

$\mathcal{P}_{l, m}^{j}\left(r_{\mathrm{c}} ; \widehat{v}_{\mathrm{c}}\right)=\frac{\left\langle\widetilde{P}_{l}^{m}(\cos \theta) w_{j, m}\left(r_{\mathrm{c}}, \theta ;{\widehat{v_{\mathrm{c}}}}_{\mathrm{c}}\right)\right\rangle_{\theta}}{\left\langle\left[w_{j, m}\left(r_{\mathrm{c}}, \theta ; \widehat{v}_{\mathrm{c}}\right)\right]^{2}\right\rangle_{\theta}}$.

Then, $\mathcal{L}_{V}^{\mathrm{AM}}$ becomes:

$$
\begin{aligned}
& \mathcal{L}_{V}^{\mathrm{AM}}= \\
& \quad \frac{1}{2} \bar{\rho}_{\mathrm{c}} r_{\mathrm{c}} \frac{1}{N_{\mathrm{c}}} \int_{\sigma} \sum_{l, m, j}\left\{\lambda_{j, m}^{1 / 2}\left(r_{\mathrm{c}} ; \widehat{v}_{\mathrm{c}}\right)\left[W_{\mathrm{CZ} ; l, m}\left(r_{\mathrm{c}} ; \sigma\right)\right]^{2}\left[\mathcal{P}_{l, m}^{j}\left(r_{\mathrm{c}} ; \widehat{v}_{\mathrm{c}}\right)\right]^{2}\right. \\
& \left.\quad \times \sin \theta\left[\widehat{\sigma}^{2} w_{j, m}\left(\mathcal{G}_{j, m}^{\varphi}-\widehat{v} \cos \theta \mathcal{G}_{j, m}^{\theta}\right)\right]_{r=r_{\mathrm{c}}} \exp \left[-\tau_{j, m}\right]\right\} \mathrm{d} \sigma,
\end{aligned}
$$

with its associated average on an isobar $\overline{\mathcal{L}_{V}^{\mathrm{AM}}}$ :

$$
\begin{aligned}
& \overline{\mathcal{L}_{V}^{\mathrm{AM}}}= \\
& \frac{3}{8} \bar{\rho}_{\mathrm{c}} r_{\mathrm{c}} \frac{1}{N_{\mathrm{c}}} \int_{\sigma}\left\{\sum_{l, m, j} \lambda_{j, m}^{1 / 2}\left(r_{\mathrm{c}} ; \widehat{v}_{\mathrm{c}}\right)\left[W_{\mathrm{CZ} ; l, m}\left(r_{\mathrm{c}} ; \sigma\right)\right]^{2}\left[\mathcal{P}_{l, m}^{j}\left(r_{\mathrm{c}} ; \widehat{v}_{\mathrm{c}}\right)\right]^{2}\right. \\
& \left.\quad \times\left\langle\sin \theta\left[\widehat{\sigma}^{2} w_{j, m}\left(\mathcal{G}_{j, m}^{\varphi}-\widehat{v} \cos \theta \mathcal{G}_{j, m}^{\theta}\right)\right]_{r=r_{\mathrm{c}}} \exp \left[-\tau_{j, m}\right]\right\rangle_{\theta}\right\} \mathrm{d} \sigma .
\end{aligned}
$$

\subsubsection{Discussion}

This search for a prescription for excitation remains major unsolved and debated question in wave-induced transport theory. To study this, different approaches have been adopted.

The first analytical one consists of deriving, using phenomenological prescritions, the energy flux transmission between the turbulent convective movements and the IGWs using the match of the wave pressure fluctuation with that of the turbulent convection. A Kolmogorov turbulent energy spectrum is assumed. This procedure is described in detail in Press (1981), Garciá López \& Spruit (1991) and Zahn et al. (1997) in the nonrotating case and by Pantillon et al. (2007) in the case where the Coriolis acceleration is taken into account.

The second semi-analytical approach consists of deriving, in the most consistent possible way, the wave amplitude by describing their stochastic volumetric excitation by the convective Reynolds stresses and the turbulent entropy advection. This method takes into account both the spatial and the temporal correlations between turbulent eddies and waves. The formalisms follow the first work by Goldreich et al. (1994) which was devoted to solar $p$-modes and adapted to IGWs by Kumar et al. (1999). These first contributions assumed a Kolmogorov energy spectrum. These works were then generalized by
Samadi et al. (2001a,b) in order to take into account a general turbulent energy spectrum which can be extracted from realistic 3-D numerical simulations of turbulent convection in stellar interiors and by Belkacem et al. (2008a,b) who derived a rigourous treatment of the excitation accounting for the non-radial character of the modes that is crucial in the case of IGWs for which the displacement is mostly horizontal. Finally, the Coriolis acceleration is now taken into account (Mathis et al. 2008; Belkacem 2008) and the generalized formalism has now to be applied to gravito-inertial waves.

Penetrative convection is also an efficient process to generate IGWs. This was first investigated by Townsend $(1965,1966)$ in the case of atmospheric flows. Then, in the stellar context, Montalbán (1994), Montalbán \& Schatzman (1996-2000), following Townsend (1966), used several models for wave excitation by plumes in order to study the problem of light element mixing induced by IGWs (see also the work by Lo \& Schatzman 1997; and Lo 1997, for the case of convective cores). However, they considered that waves are generated solely by turbulence inside plumes and they did not investigate the generation of waves caused by the impact of plumes on the stably stratified region that has been undertaken (cf. Belkacem 2008).

The major approach to obtain prescriptions for the wave energy spectrum in this case consists of computing numerical simulations of turbulent penetrative convection at the interface between convective and radiative regions. Such simulations have shown IGW excitation (see for example Hurlburt et al. 1986, 1994; Andersen 1994; Brummell et al. 2002; Browning et al. 2004; Rogers \& Glatzmaier 2005; Rogers et al. 2006) but specific work has to be undertaken to provide a quantitative estimate of the amplitude and of the spectrum of waves.

Initial work dedicated to such a study has been completed in 2-D Cartesian geometry by Kiraga et al. (2003). In this work, the assumed stratification is polytropic and the authors add a viscous boundary layer at the bottom of the stable zone in order to avoid the reflexion of excited waves and thus the appearance of normal modes in the simulation box. Their main results are that phenomenological semi-analytical models (the Garcià-Lopez \& Spruit one, hereafter GLS91; or the plume model by Rieutord \& Zahn 1995, hereafter RZ95) significantly underestimate the flux of IGWs by a factor of 100 (GLS91) and 10 (RZ95) compared to 2-D direct numerical simulations. On the other hand, in the domain $\left(\sigma, k_{H}\right)$, the numerically obtained wave spectrum is much broader than those predicted using GLS91 which results in a lack of high frequency waves and RZ95 where low frequencies are missing. However, the authors emphasized that 2-D simulations probably produce stronger downflows compared to more realistic 3-D simulations. This is the reason why Kiraga et al. (2005) revisited their own work comparing their previous results with those obtained in a 3-D Cartesian box using the same stratification where downdrafts are significantly less vigorous. On one hand, the excited IGWs have lower amplitude. On the other hand, the wave energy flux increases with the depth of the convective layer.

In the same way, Dintrans et al. (2005) proposed a quantitative investigation of the spectrum, the amplitude and the lifetime of IGWs excited by penetrative convection in solar-like stars using 2-D numerical simulations of compressible convection assuming that the gas is monoatomic and perfect. The wave generation is studied from the linear response of the radiative zone to the plume penetration using projections onto the $g$-mode linear eigenfunctions. The authors show that up to $40 \%$ of the total kinetic energy is transmitted to IGWs during times of significant excitation. 
Finally, work is now undertaken to take into account realistic stratification, the global geometry, and the (differential) rotation. In this way, Rogers \& Glatzmaier (2005b; and Rogers \& Glatzmaier 2006) computed integrated models of the Sun interior (both the convective envelope and the radiative core) in 2-D polar geometry that represents the equatorial plane of the Sun using a realistic stratification given by a solar model. As in the work by Kiraga et al. (2003), the frequency spectrum found is broader than those determined using semi-analytical models with a more uniform distribution between low and high frequencies. On the other hand, it is shown that non-linear effects have to be taken into account. These effects broaden the frequency ridges in the dispersion relation. Furthermore, just under the convection zone, the energy is increased by two orders of magnitude over what the linear dispersion relation would predict for energy in waves. Work on such numerical simulations is now in progress in 3-D spherical geometry with using the Anelastic Spherical Harmonics code (see Clune et al. 1999; Brun et al. 2004, for the code description; and Brun 2009).

Therefore, all these possible sources of prescription for the wave excited spectrum have to be carefully examined given its uncertainty; this will be studied in the application of our formalism.

\subsection{Transport of angular momentum}

Due to the structure of the equation for the transport of angular momentum given in Eq. (105), we follow the procedure adopted in Zahn (1992) and in Mathis \& Zahn (2004, 2005). Therefore, the angular velocity is expanded as follows

$\Omega(r, \theta)=\bar{\Omega}(r)+\widehat{\Omega}(r, \theta)$.

$\bar{\Omega}(r)$ and $\widehat{\Omega}(r, \theta)$ are respectively the mean rotation rate on the isobar and its fluctuation. Moreover, it is assumed that $\widehat{\Omega} \ll \bar{\Omega}$ that is enforced by a stronger turbulent transport in the horizontal directions than in the radial one. This behaviour of the turbulence is due to the stable stratification of stellar radiation zones that inhibits the radial turbulent movements. Thus, the horizontal turbulent viscosity, $v_{H}$, is greater than the radial one, $v_{V}$. The associated horizontal gradient of angular velocity is thus weaker than the radial one. This is the approximation of the so-called "shellular rotation". $\bar{\Omega}$ and $\widehat{\Omega}$ are respectively defined and expanded as

$\bar{\Omega}(r)=\frac{\left\langle\sin ^{2} \theta \Omega(r, \theta)\right\rangle_{\theta}}{\left\langle\sin ^{2} \theta\right\rangle_{\theta}}$ and $\widehat{\Omega}(r, \theta)=\sum_{l>0} \Omega_{l}(r) Q_{l}(\theta),($

where the $\Omega_{l}$ are the radial modal functions of the horizontal differential rotation. Due to the mathematical properties of Eq. (105) and to the definition of $\bar{\Omega}$, the special angular functions $Q_{l}(\theta)$ are defined by (see Mathis \& Zahn 2004, for a detailed discussion):

$Q_{l}(\theta)=P_{l}(\cos \theta)-I_{l}$ with $I_{l}=\frac{\left\langle\sin ^{2} \theta P_{l}(\cos \theta)\right\rangle_{\theta}}{\left\langle\sin ^{2} \theta\right\rangle_{\theta}}$.

On the other hand, we recall that the meridional circulation is expanded in vectorial spherical harmonics:

$\mathcal{U}_{M}(r, \theta)=\sum_{l>0}\left\{U_{l}(r) P_{l}(\cos \theta) \widehat{\mathbf{e}}_{r}+V_{l}(r) \frac{\mathrm{d} P_{l}(\cos \theta)}{\mathrm{d} \theta} \widehat{\mathbf{e}}_{\theta}\right\}$,
$U_{l}$ and $V_{l}$ being the radial modal functions respectively in the radial direction and in the latitudinal one. The circulation is also an anelastic flow such that $\boldsymbol{\nabla} \cdot\left(\bar{\rho} \boldsymbol{U}_{\mathrm{M}}\right)=0$ that leads to the following relation between $U_{l}$ and $V_{l}$ :

$V_{l}=\frac{1}{l(l+1) \bar{\rho} r} \frac{\mathrm{d}}{\mathrm{d} r}\left(\bar{\rho} r^{2} U_{l}\right)$.

The definitions being given, we now have to derive the respective evolution equations for $\bar{\Omega}$ and $\Omega_{l}$.

\subsubsection{Transport of the mean differential rotation}

Waves deposit their angular momentum in stellar radiation zones as they are damped. The total local action of angular momentum is given by

$\mathcal{L}_{V ; j, m}^{\mathrm{AM}}(r, x ; \widehat{v})=\int_{\sigma}\left\{\mathcal{L}_{V ; j, m}^{\mathrm{AM}}\left(r_{\mathrm{c}}, x ; \widehat{v}_{\mathrm{c}}\right) \exp \left[-\tau_{j, m}\right]\right\} \mathrm{d} \sigma$.

The induced transport of angular momentum by IGWs is then ruled by the radial derivative of this action of angular momentum:

$$
\left[\bar{\rho} \frac{\mathrm{d}}{\mathrm{d} t}\left(r^{2} \bar{\Omega}\right)\right]_{\mathrm{IGWs}}=-\frac{1}{r^{2}} \partial_{r}\left[\overline{\mathcal{L}_{V}^{\mathrm{AM}}}(r)\right] .
$$

Let us first look at the damping integral given in Eq. (68) and assume that both prograde and retrograde waves are excited with the same amplitude and have the same eigenvalue $\left(\lambda_{j, m}\right)$. In solidbody rotation, both waves are equally dissipated when travelling inward and there is no impact on the distribution of angular momentum. In the presence of differential rotation, the situation is different. If the interior is rotating faster than the convection zone, the local frequency of prograde waves decreases, which enhances their dissipation; the corresponding retrograde waves are then dissipated further inside. This produces an increase of the local differential rotation and creates a doubled-peaked shear layer because positive and negative local shears are amplified by prograde and retrograde waves and even a small perturbation can trigger this (the prograde waves transport a positive flux of angular momentum and the retrograde waves a negative one). In the presence of shear turbulence, this layer can oscillate (this depends on the vertical eddy-viscosity value), producing a Shear Layer Oscillation (S.L.O.) (cf. Ringot 1998; Kumar et al. 1999; Kim \& MacGregor 2001; Talon \& Charbonnel 2005). This is the first important feature of wave-mean flow interaction.

This SLO acts as a filter through which most low-frequency waves cannot pass. However, if the core is rotating faster than the surface, this filter is not quite symmetric, and retrograde waves will be favored. As a result, a net negative flux of angular momentum will produce a spin down of the core (Talon et al. 2002) and this is the filtered mean angular momentum action, $\overline{\mathcal{L}_{V}^{\mathrm{AM}} \text {,fil }}(r)$, that contributes to the secular evolution of angular momentum (for details, see Talon \& Charbonnel 2005). This may play a key role in flattening the rotation profile as observed in the present Sun (Charbonnel \& Talon 2005).

Then, averaging Eq. (105) on the isobar and using the assumption that $\bar{\Omega} \gg \Omega_{l}$, the transport equation for the mean differential rotation is obtained:

$$
\begin{aligned}
\bar{\rho} \frac{\mathrm{d}}{\mathrm{d} t}\left(r^{2} \bar{\Omega}\right)- & \frac{1}{5 r^{2}} \partial_{r}\left(\bar{\rho} r^{4} \bar{\Omega} U_{2}\right)= \\
& \frac{1}{r^{2}} \partial_{r}\left(\bar{\rho} \nu_{V} r^{4} \partial_{r} \bar{\Omega}\right)-\frac{1}{r^{2}} \partial_{r}\left[\overline{\mathcal{L}_{V}^{\mathrm{AM}, \mathrm{fil}}}\right] .
\end{aligned}
$$


The left-hand side is the sum of the temporal Lagrangian variation of the mean vertical angular momentum (we recall that $\frac{\mathrm{d}}{\mathrm{d} t}=\partial_{t}+\dot{r} \partial_{r}$ is the time-Lagrangian derivative) and of the divergence of the mean flux of angular momentum advected by the meridional circulation. The right-hand side corresponds to the sum of the ones of the radial turbulent viscous flux and of the flux transported by the Reynolds stresses associated with the waves.

Defining the respective radius $r_{b}$ and $r_{t}$ of the positions of the lower and of the upper border of the radiation region, the boundary conditions for Eq. (148), which is a fourth-order equation in $\bar{\Omega}$ (see Zahn 1992; Spiegel \& Zahn 1992; Maeder \& Zahn 1998; Mathis \& Zahn 2004), are given by:

$$
\frac{\mathrm{d}}{\mathrm{d} t}\left[\int_{0}^{r_{b}} r^{\prime}{ }^{4} \bar{\rho} \bar{\Omega}_{\mathrm{CZ}} \mathrm{d} r^{\prime}\right]=\frac{1}{5} r^{4} \bar{\rho} \bar{\Omega} U_{2}+\bar{\rho} v_{V} r^{4} \partial_{r} \bar{\Omega}-\overline{\mathcal{L}_{V}^{\mathrm{AM}, \mathrm{fil}}}
$$

at $r=r_{b}$,

$\frac{\mathrm{d}}{\mathrm{d} t}\left[\int_{r_{t}}^{R} r^{\prime}{ }^{4} \bar{\rho} \bar{\Omega}_{\mathrm{CZ}} \mathrm{d} r^{\prime}\right]=-\frac{1}{5} r^{4} \bar{\rho} \bar{\Omega} U_{2}-\bar{\rho} \nu_{V} r^{4} \partial_{r} \bar{\Omega}+\overline{\mathcal{L}_{V}^{\mathrm{AM}, \mathrm{fil}}}-\mathcal{F}_{\Omega}$

at $r=r_{t}$ and

$\partial_{r} \bar{\Omega}=\partial_{r} \bar{\Omega}_{\mathrm{CZ}}$ both at $r=r_{b}$ and $r_{t}$,

where we have defined:

$\bar{\Omega}_{\mathrm{CZ}}(r)=\frac{\left\langle\sin ^{2} \theta \Omega_{\mathrm{CZ}}\right\rangle_{\theta}}{\left\langle\sin ^{2} \theta\right\rangle_{\theta}}$.

$\mathcal{F}_{\Omega}$ is the flux of angular momentum loss at the surface at $r=R$, where $R$ is the radius of the star. It is driven by magnetic winds in the case of solar-type stars (cf. Schatzman 1962) and by radiative ones in the case of massive stars (cf. Maeder 1999).

This equation has been implemented in stellar evolution codes in simplified cases to study the wave-induced effects on the rotational transport (Talon \& Charbonnel 2005; Pantillon et al. 2007; Mathis et al. 2008).

The formalism presented here takes into account the latitudinal but also the radial strong gradients of angular velocity that may develop during stellar evolution, as could be the case in the vertical direction when the extraction of angular momentum occurs in the bulk of stellar radiation zones (cf. Talon \& Charbonnel 2005). In the case of such strong radial variation of the rotation rate, the simplest formalism of the "weak differential rotation" does not apply any longer and the general one, which is derived here, will be adopted.

On the other hand, in the case of general differential rotation, it is always possible to study the mean vertical transport of angular momentum, Eq. (148) becoming

$$
\begin{aligned}
& \bar{\rho} \frac{\mathrm{d}}{\mathrm{d} t}\left(r^{2} \bar{\Omega}\right)+\frac{3}{4}\langle\boldsymbol{\nabla} \cdot[\left.\left.\bar{\rho} r^{2} \sin ^{2} \theta \Omega \mathcal{U}_{M}(r, \theta)\right]\right\rangle_{\theta}= \\
& \frac{1}{r^{2}} \partial_{r}\left(\bar{\rho} v_{V} r^{4} \partial_{r} \bar{\Omega}\right)-\frac{1}{r^{2}} \partial_{r}\left[\overline{\mathcal{L}_{V}^{\mathrm{AM}, \text { fil }}}(r)\right] .
\end{aligned}
$$

The case of horizontal differential rotation will be discussed at the end of the next section.

\subsubsection{Transport of horizontal differential rotation}

In this section, the goal is to derive the equation which governs the transport of the horizontal differential rotation $\widehat{\Omega}$. To achieve this aim, the procedure developped in Mathis \& Zahn (2005) to treat the impact of a mean-axisymmetric magnetic field on the rotational transport is adopted.

First, the action of angular momentum of the waves $\mathcal{L}_{V}^{\mathrm{AM}}$ is expanded in the Legendre polynomials $P_{l}$ as has been done in Mathis \& Zahn (2005) for the Lorentz torque $\Gamma_{\mathcal{F}_{\mathcal{L}}}(r, \theta)$ (cf. Eq. (47) in this paper):

$\mathcal{L}_{V}^{\mathrm{AM}}(r, \theta)=\sum_{l=0}^{\infty} \mathcal{L}_{V ; l}^{\mathrm{AM}}(r) \sin ^{2} \theta P_{l}(\cos \theta)$

Using its expression given in Eqs. (127-132), the radial functions $\mathcal{L}_{V ; l}^{\mathrm{AM}}$ are obained:

$\mathcal{L}_{V ; l}^{\mathrm{AM}}(r)=-r_{\mathrm{c}}^{2} \int_{\sigma} \sum_{m, j} \mathcal{A}_{j, m}^{l}(r) \mathrm{d} \sigma$

where

$$
\begin{aligned}
& \mathcal{A}_{j, m}^{l}(r)=\frac{1}{\left\langle\left[P_{l}(\cos \theta)\right]^{2}\right\rangle_{\theta}} \\
& \quad \times\left\langle(\sin \theta)^{-2} \frac{\widehat{m}\left(r_{\mathrm{c}}, \theta ; \widehat{v}_{\mathrm{c}}\right)}{\widehat{\sigma}_{\mathrm{CZ}}} \mathcal{F}_{V ; j, m}^{\mathrm{E}}\left(r_{\mathrm{c}}, \theta ; \widehat{v}_{\mathrm{c}}\right) \exp \left[-\tau_{j, m}\right] P_{l}(\cos \theta)\right\rangle_{\theta}
\end{aligned}
$$

and

$$
\begin{aligned}
\mathcal{L}_{V ; l}^{\mathrm{AM}}(r)= & \frac{1}{2} \bar{\rho}_{\mathrm{c}} r_{\mathrm{c}} \frac{1}{N_{\mathrm{c}}} \int_{\sigma}\left\{\sum_{m, j} \lambda_{j, m}^{1 / 2}\left(r_{\mathrm{c}} ; \widehat{v}_{\mathrm{c}}\right) a_{j, m}^{2}\left(r_{\mathrm{c}}\right)\right. \\
& \left.\times \frac{\left\langle\mathcal{B}_{j, m}(r, \theta) P_{l}(\cos \theta)\right\rangle_{\theta}}{\left\langle\left[P_{l}(\cos \theta)\right]^{2}\right\rangle_{\theta}}\right\} \mathrm{d} \sigma
\end{aligned}
$$

where

$$
\begin{aligned}
\mathcal{B}_{j, m}(r, \theta)= & {\left[\widehat{\sigma}^{2}(\sin \theta)^{-1} w_{j, m}\left(\mathcal{G}_{j, m}^{\varphi}-\widehat{v} \cos \theta \mathcal{G}_{j, m}^{\theta}\right)\right]_{r=r_{\mathrm{c}}} } \\
& \times \exp \left[-\tau_{j, m}\right] .
\end{aligned}
$$

We establish the equation governing the horizontal transport of angular momentum by multiplying Eq. (148) by $\sin ^{2} \theta$ and subtracting it from its bidimensional original form given in Eq. (105):

$$
\begin{aligned}
\bar{\rho} & \frac{\mathrm{d}}{\mathrm{d} t}\left(r^{2} \sin ^{2} \theta \widehat{\Omega}\right)+\nabla \cdot\left(\bar{\rho} r^{2} \sin ^{2} \theta \bar{\Omega} \mathcal{U}_{\mathrm{M}}\right) \\
& +\frac{\sin ^{2} \theta}{5 r^{2}} \partial_{r}\left(\bar{\rho} r^{4} \bar{\Omega} U_{2}\right)=\frac{\sin ^{2} \theta}{r^{2}} \partial_{r}\left(\bar{\rho} v_{V} r^{4} \partial_{r} \widehat{\Omega}\right) \\
& +\frac{1}{\sin \theta} \partial_{\theta}\left(\bar{\rho} v_{H} \sin ^{3} \theta \partial_{\theta} \widehat{\Omega}\right)-\frac{1}{r^{2}} \partial_{r}\left[\mathcal{L}_{V}^{\mathrm{AM}}-\sin ^{2} \theta \overline{\mathcal{L}_{V}^{\mathrm{AM}}}\right]
\end{aligned}
$$

The fluctuation $\widehat{\Omega}$ again has been neglected compared to the mean $\bar{\Omega}$ in the advection term. Next, we replace $\widehat{\Omega}$ by its expansion given in Eq. (142). For $l=2$, this equation separates into

$$
\left[\bar{\rho} \frac{\mathrm{d}}{\mathrm{d} t}\left(r^{2} \Omega_{2}\right)\right]_{\mathrm{IGWs}}=-\frac{1}{r^{2}} \partial_{r}\left[\mathcal{L}_{V ; 2}^{\mathrm{AM}}(r)\right]
$$

for the SLO part, and

$$
\begin{aligned}
& \bar{\rho} \frac{\mathrm{d}}{\mathrm{d} t}\left(r^{2} \Omega_{2}\right)-2 \bar{\rho} \bar{\Omega} r\left[2 V_{2}-\alpha(r) U_{2}\right]= \\
& \frac{1}{r^{2}} \partial_{r}\left(\bar{\rho} v_{V} r^{4} \partial_{r} \Omega_{2}\right)-10 \bar{\rho} v_{H} \Omega_{2}-\frac{1}{r^{2}} \partial_{r}\left[\mathcal{L}_{V ; 2}^{\mathrm{AM}, \mathrm{fil}}(r)\right]
\end{aligned}
$$


for the secular one, where $\mathcal{L}_{V ; 2}^{\mathrm{AM} \text {,fil }}$ is the filtered horizontal action of angular momentum and

$\alpha=\frac{1}{2} \frac{\mathrm{d} \ln \left(r^{2} \bar{\Omega}\right)}{\mathrm{d} \ln r}$.

This can be simplified by assuming that the turbulent transport is much more efficient in the horizontal direction than in the radial one (i.e. $v_{V} \ll v_{H}$ ):

$$
\begin{aligned}
\bar{\rho} \frac{\mathrm{d}}{\mathrm{d} t}\left(r^{2} \Omega_{2}\right) & -2 \bar{\rho} \bar{\Omega} r\left[2 V_{2}-\alpha U_{2}\right]= \\
& -10 \bar{\rho} v_{H} \Omega_{2}-\frac{1}{r^{2}} \partial_{r}\left[\mathcal{L}_{V ; 2}^{\mathrm{AM}, \mathrm{fil}}(r)\right] .
\end{aligned}
$$

In the asymptotic regime, where $t \gg r^{2} / v_{H}$, a stationary state can be reached:

$v_{H} \Omega_{2}=\frac{1}{5} r\left[2 V_{2}-\alpha U_{2}\right] \bar{\Omega}-\frac{1}{10 \bar{\rho}} \frac{1}{r^{2}} \partial_{r}\left[\mathcal{L}_{V ; 2}^{\mathrm{AM}, \mathrm{fil}}(r)\right]$,

where the horizontal turbulent diffusion balances the horizontal advection and the Reynolds stresses of the waves.

For $l>2$, the situation is intricate, because of couplings between terms of different $l$ in $\mathcal{L}_{V ; l}^{\mathrm{AM}}$ that prevent a clean separation for them.

Therefore, as a first step, we choose here to stop the expansion of the angular velocity at $\Omega_{2}$. This means that we assume a low resolution in latitude which is valid only as long as the latitudinal differential rotation $(\widehat{\Omega})$ is a linear perturbation of the mean rotation rate on the isobar $(\bar{\Omega})$ and can be described correctly by the first horizontal function $\left(Q_{2}(\theta)\right)$, this situation being enforced by the strong horizontal turbulent transport. However, care must be taken in the cases where a more refined latitudinal resolution is needed or where the horizontal differential rotation becomes stronger. In the first case, supplementary modes $(l>2)$ have to be taken into account, while in the second one, the bidimensional original equation for the transport of the angular momentum (Eq. (105)) has to be solved directly using Eqs. (116) and (132). This could be achieved numerically or using a semianalytical treatment such as those developed by Spiegel \& Zahn (1992).

For the boundary conditions, we assume that there are no stresses between the radiative and the the convective zones that leads to:

$\Omega_{2}=\Omega_{\mathrm{CZ} ; 2}$ both at $r=r_{b}$ and $r_{t}$

and

$\partial_{r} \Omega_{2}=\partial_{r} \Omega_{\mathrm{CZ} ; 2}$ both at $r=r_{b}$ and $r_{t}$,

where the angular velocity inside the convective zone $\Omega_{\mathrm{CZ}}$ has been expanded as in Eqs. (141) and (142).

Equation (159) allows us to study the effect of the waves on the transport of angular momentum in the latitudinal direction during stellar evolution. This is an important point with respect to the aim we have to study to the first order the secular effects of tachocline(s) on stellar evolution in a consistent way (see Brun et al. 1999; Mathis \& Zahn 2004). Moreover, this is a key point since tachocline(s) are precisely the seat of the stochastic excitation of the low-frequency waves that are transporting angular momentum. This also opens the field for a bidimensional study to draw a coherent picture of the dynamics of the Shear Layer Oscillation due to the high degree waves that have a strong effect on the deeper transport (see Kim \& MacGregor 2001-2003;
Talon \& Charbonnel 2005) and that also could be the cause of a non-magnetic cyclic solar and stellar activity (see Dzhalilov et al. 2002; and Dzhalilov \& Straude 2004; Turck-Chièze \& Talon 2008).

\section{Conclusion and perspectives}

In this work, we present a complete formalism allowing us to treat the action of a general strong differential rotation on lowfrequency waves in stellar radiation zones and their feed-back on the angular momentum transport. As has been shown in Sect. 3.4.6, the traditional and the JWKB approximations can be adopted for regular elliptic gravito-inertial waves such that $\sigma \ll N$ and $\mathcal{D}>0$ while $2 \Omega \ll N$. This allows us to analytically derive the spatial structure of their pressure fluctuation and velocity field. The results have been compared to those that have been obtained in the "weak differential rotation" case where the rotation is almost a solid body-one plus a residual radial differential rotation.Next, the transport equations for respectively the mean differential rotation and the latitudinal one have been derived in a form which allows a direct implementation in dynamical stellar evolution codes (see Talon \& Charbonnel 2005).

In the near future, the hyperbolic regime, where the traditional approximation fails, and the potential effect of the corotation resonance have to be carefully studied, particulary in the context of the deposition and extraction of angular momentum where the Coriolis acceleration modifies both the damping and the excitation rates.

The astrophysical applications of this work are discussed in Talon (2007) and references therein.

Finally, these results have to be generalized and applied to the cases of stellar and planetary tides.

In a forthcoming paper (Paper II), this formalism will be applied to the case of the solar differential rotation.

Acknowledgements. I thank the referee for her remarks and suggestions that improved and clarified the original manuscript. I am also grateful to K. Belkacem, S. Turck-Chièze and J.-P. Zahn who kindly commented on the manuscript and suggested improvements. Finally, I thank S. Talon for fruitful discussions. This work was supported in part by the Programme National de Physique Stellaire.

\section{References}

Abramowitz, M., \& Stegun, I. 1972, Handbook of mathematical functions (New York: Dover)

Aerts, C., Christensen-Dalsgaard, J., Cunha, M., \& Kurtz, D. W. 2008, Sol. Phys., 251, 3

Andersen, B. N. 1994, Sol. Phys., 152, 241

Ando, H. 1985, PASJ, 37, 47

Barnes, G., MacGregor, K. B., \& Charbonneau, P. 1998, ApJ, 498, L169

Belkacem, K. 2008, Ph.D. Thesis, Observatoire de Paris

Belkacem, K., Samadi, R., Goupil, M.-J., \& Dupret, M.-A. 2008, A\&A, 478, 163

Belkacem, K., Samadi, R., Goupil, M.-J., et al. 2009, A\&A, 494, 191

Berthomieu, G., Gonczi, G., Graff, Ph., Provost, J., \& Rocca, A. 1978, A\&A, 70, 597

Bildsten, L., Ushomirsky, G., \& Cutler, C. 1996, ApJ, 460, 827

Braithwaite, J. 2006, A\&A, 449, 451

Braithwaite, J., \& Spruit, H. C. 2005, Nature, 431, 819

Browning, M. K., Brun, A. S., \& Toomre, J. 2004, ApJ, 601, 512

Brummell, N. H., Clune, T. L., \& Toomre, J. 2002, ApJ, 570, 825

Brun, A. S., \& Toomre, J. 2002, ApJ, 570, 865

Brun, A. S., \& Zahn, J.-P. 2006, A\&A, 457, 665

Brun, A. S., Turck-Chièze, S., \& Zahn, J.-P. 1999, ApJ, 525, 1032

Brun, A. S., Miesch, M., \& Toomre, J. 2004, ApJ, 614, 1073

Bretherton, F. P. 1969, Quat. J. Roy. Met. Soc., 95, 213

Busse, F. H. 1982, ApJ, 259, 759

Charbonneau, P., \& MacGregor, K. B. 1993, ApJ, 417, 762

Charbonnel, C., \& Talon, S. 2005, Science, 309, 2189

Clune, T. L., et al. 1999, Parallel Comput., 25, 361 
Cowling, T. G. 1941, MNRAS, 101, 367

Decressin, T., Mathis, S., Palacios, A., et al. 2009, A\&A, 495, 271

Dintrans, B. 1999, Ph.D. Thesis, Université Toulouse III

Dintrans, B., \& Brandenburg, A. 2004, A\&A, 421, 775

Dintrans, B., \& Rieutord, M. 2000, A\&A, 354, 86

Dintrans, B., Rieutord, M., \& Valdettaro, L. 1999, J. Fluid Mech., 398, 271

Dintrans, B., Brandenburg, A., Nordlund, A., \& Stein, R. F. 2005, A\&A, 438, 365

Dzhalilov, N. S., \& Staude, J. 2004, A\&A, 421, 305

Dzhalilov, N. S., Staude, J., \& Oraevsky, V. N. 2002, A\&A, 384, 282

Eckart, C. 1960, Hydrodynamics of Oceans and Atmospheres (Oxford: Pergamon Press)

Eggenberger, P., Maeder, A., \& Meynet, G. 2005, A\&A, 440, L9

Espinosa Lara, F., \& Rieutord, M. 2007, A\&A, 470, 1013

Friedlander, S. 1987, Geophys. J. Roy. Astr. Soc., 89, 637

Friedlander, S., \& Siegmann, W. L. 1982, Geophys. Astrophys. Fluid Dynamics, 19,267

Fröman, N., \& Fröman, P. O. 1965, JWKB Approximation: Contributions to the Theory (Amsterdam: North-Holland Publishing Company)

García, R. A., Turck-Chièze, S., Jiménez-Reyes, S. J., et al. 2007, Science, 316, 1591

García-López, R. J., \& Spruit, H. C. 1991, ApJ, 377, 268

Garaud, P. 2001, MNRAS, 324, 68

Garaud, P. 2002a, MNRAS, 329, 1

Garaud, P. 2002b, MNRAS, 335, 707

Gerkema, T., \& Shrira, V. I. 2005, J. Fluid Mech., 529, 195

Gerkema, T., Zimmerman, J. T. F., Mass, L. R. M., \& Van Haren, H. 2008, Rev. Geophys., 46, CiteID RG2004

Goldreich, P., \& Tremaine, S. 1979, ApJ, 233, 857

Goldreich, P., \& Nicholson, P. D. 1989a, ApJ, 342, 1075

Goldreich, P., \& Nicholson, P. D. 1989b, ApJ, 342, 1079

Goldreich, P., Murray, N., \& Kumar, P. 1994, ApJ, 424, 466

Gough, D. O., \& McIntyre, M. E. 1998, Nature, 394, 567

Hayes, W. D. 1970, Proc. Roy. Soc. London A, 320, 187

Hough, S. S. 1898, Phil. Trans. Roy. Soc. A, 191, 139

Hurlburt, N. E., Toomre, J., \& Massaguer, J. M. 1986, ApJ, 311, 563

Hurlburt, N. E., Toomre, J., Massaguer, J. M., \& Zahn, J.-P. 1994, ApJ, 421, 245

Kim, E. J., \& MacGregor, K. B. 2001, ApJ, 556, L117

Kim, E. J., \& MacGregor, K. B. 2003, ApJ, 588, 645

Kiraga, M., Stepien, K., \& Jahn, K. 2005, Acta Astron., 55, 205

Kiraga, M., Jahn, K., Stepien, K., \& Zahn, J.-P. 2003, Acta Astron., 53, 321

Kumar, P., \& Quataert, E. J. 1997, ApJ, 475, L143

Kumar, P., Talon, S., \& Zahn, J.-P. 1999, ApJ, 520, 859

Landau, L. D., \& Lifchitz, E. M. 1966, Theoretical Physics: Quantum Mechanics (Moscow: Mir)

Laplace, P.-S. 1799, Mécanique Céleste, Bureau des Longitudes, Paris

Lee, U., \& Saio, H. 1997, ApJ, 491, 839

Lighthill, J. 1978, Waves in fluids (Cambridge: Cambridge University Press)

Lindzen, R. S., \& Chapman, S. 1969, Space Sci. Rev., 10, 3

Lo, Y.-C. 1997, Ph.D. Thesis, Université Louis Pasteur - Observatoire de Strasbourg

Lo, Y.-C., \& Schatzman, E. 1997, A\&A, 322, 545

Longuet-Higgins, F. R. S. 1968, Phil. Trans. Roy. Soc. A, 262, 511

Maeder, A. 1999, A\&A, 347, 185

Maeder, A. 2003, A\&A, 399, 263

Maeder, A., \& Meynet, G. 2004, A\&A, 422, 225

Maeder, A., \& Zahn, J.-P. 1998, A\&A, 334, 1000

Mathis, S. 2005, Ph.D. Thesis, Université Paris XI

Mathis, S., \& Zahn, J.-P. 2004, A\&A, 425, 229

Mathis, S., \& Zahn, J.-P. 2005, A\&A, 440, 653

Mathis, S., Palacios, A., \& Zahn, J.-P. 2004, A\&A, 425, 243

Mathis, S., Decressin, T., Palacios, A., et al. 2007, Astron. Nachr., 328, 1062

Mathis, S., Talon, S., Pantillon, F.-P., \& Zahn, J.-P. 2008, Sol. Phys., 251, 101

Mathis, S., Belkacem, K., Goupil, M.-J., \& Samadi, R. 2009, Commun. Asteroseismol., 157, 144

Mathur, S., Eff-Darwich, A., García, R. A., \& Turck-Chièze, S. 2008, A\&A, 484, 517

Menou, K., Balbus, S. A., \& Spruit, H. C. 2004, ApJ, 607, 564

Meynet, G., \& Maeder, A. 2000, A\&A, 361, 101

Miles, J. W. 1974, J. Fluid Mech., 66, 241
Miles, J. W. 1977, Proc. Roy. Soc. London A, 353, 377

Montalbán, J. 1994, A\&A, 281, 421

Montalbán, J., \& Schatzman, E. 1996, A\&A, 305, 513

Montalbán, J., \& Schatzman, E. 2000, A\&A, 354, 943

Nicholson, P. D. 1989, Ph.D. Thesis, Caltech

Ogilvie, G. I., \& Lin, D. N. C. 2004, ApJ, 610, 477

Palacios, A., Talon, S., Charbonnel, C., \& Forestini, M. 2003, A\&A, 399, 603

Palacios, A., Charbonnel, C., Talon, S., \& Siess, L. 2006, A\&A, 453, 261

Papaloizou, J. C. B., \& Savonije, G. J. 1997, MNRAS, 291, 651

Pantillon, F. P., Talon, S., \& Charbonnel, C. 2007, A\&A, 474, 155

Pedlosky, J. 1987, Geophysical Fluid Dynamics, second edition (New York: Springer)

Press, W. H. 1981, ApJ, 245, 286

Rieutord, M. 2006, A\&A, 451, 1025

Rieutord, M., \& Zahn, J.-P. 1995, A\&A, 296, 127

Ringot, O. 1998, A\&A, 335, L89

Rogers, T. M., \& Glatzmaier, G. A. 2005a, ApJ, 620, 432

Rogers, T. M., \& Glatzmaier, G. A. 2005b, MNRAS, 364, 1135

Rogers, T. M., \& Glatzmaier, G. A. 2006, ApJ, 653, 756

Rogers, T. M., Glatzmaier, G. A., \& Jones, C. A. 2006, ApJ, 653, 765

Rogers, T. M., MacGregor, K. B., \& Glatzmaier, G. A. 2008, MNRAS, 387, 616

Samadi, R., \& Goupil, M.-J. 2001a, A\&A, 370, 136

Samadi, R., Goupil, M.-J., \& Lebreton, Y. 2001b, A\&A, 370, 147

Savonije, G. J., \& Witte, M. G. 2002, A\&A, 386, 211

Schatzman, E. 1962, Ann. Astrophys., 25, 18

Schatzman, E. 1993, A\&A, 279, 431

Schatzman, E. 1993, A\&A, 271, L29

Spiegel, E. A., \& Zahn, J.-P. 1992, A\&A, 265, 106

Spruit, H. C. 1999, A\&A, 349, 189

Spruit, H. C. 2002, A\&A, 381, 923

Stewartson, K., \& Richard, J. 1969, J. Fluid Mech., 35, 759

Stewartson, K., \& Walton, I. C. 1976, Proc. Roy. Soc. London A, 349, 141

Stein, R. F., \& Nordlund, A. 1998, ApJ, 499, 914

Talon, S. 1997, Ph.D. Thesis, Université Paris VII

Talon, S. 2008, Transport processes in stars: diffusion, rotation, magnetic fields and internal waves in Stellar Nucleosynthesis: 50 years after BBFH, ed. C. Charbonnel, \& J.-P. Zahn, EAS Pub. Ser., 32, 81

Talon, S., \& Charbonnel, C. 2005, A\&A, 440, 981

Talon, S., \& Zahn, J.-P. 1997, A\&A, 317, 749

Talon, S., Zahn, J.-P, Maeder, A., \& Meynet, G. 1997, A\&A, 322, 209

Talon, S., Kumar, P., \& Zahn, J.-P. 2002, ApJ, 574, L175

Thompson, M. J., Christensen-Dalsgaard, J., Miesch, M. S., \& Toomre, J. 2003, ARA\&A, 41, 599

Turck-Chièze, S., \& Talon, S. 2008, Adv. Space Res., 41, 855

Turck-Chièze, S., García, R. A., Couvidat, S., et al. 2004, ApJ, 604, 455

Turck-Chièze, S., Appourchaux, T., Ballot, J., et al. 2005, Trends in Space Science and Cosmic Vision 2020-39th ESLAB Symposium, ed. F. Favata, J. Sanz-Forcada, A. Gimenez, \& B. Battrick, ESA SP, 588, 193

Turck-Chièze, S., Carton, P.-H., Ballot, J., et al. 2006, Adv. Space Res., 38, 1812

Turck-Chièze, S., Carton, P. H., Mathur, S., et al. 2008, Astron. Nachr., 329, 521

Turck-Chièze, S., et al. 2008, Exp. Astron., special volume Cosmic Vision, ESA, in press

Townsend, A. A. 1965, J. Fluid Mechanics, 22, 241

Townsend, A. A. 1966, J. Fluid Mechanics, 24, 307

Turner, J. S. 1986, J. Fluid Mechanics, 173, 431

Unno, W., Osaki, Y., Ando, H., Saio, H., \& Shibahashi, H. 1989, Non-radial oscillations of stars, second edition (Tokyo: University of Tokyo Press)

Vallée, O., \& Soares, M. 1998, Airy's functions for Physics (Paris, New York: Diderot éditeur, arts et sciences)

Young, P. A., Knierman, K. A., Rigby, J. R., \& Arnett, D. 2003, ApJ, 595, 1114 Zahn, J.-P. 1966, Annales d'Astrophysique, 29, 313

Zahn, J.-P. 1975, A\&A, 41, 329

Zahn, J.-P. 1983, Saas-Fee Advanced Course 13, Astrophysical Processes in Upper Main Sequence Stars, ed. B. Hauck, \& A. Maeder (Geneva Observatory), 253

Zahn, J.-P. 1992, A\&A, 265, 115

Zahn, J.-P. 2005, EAS Publ. Ser., 17, 157

Zahn, J.-P., Talon, S., \& Matias, J. 1997, A\&A, 322, 320

Zahn, J.-P., Brun, A. S., \& Mathis, S. 2007, A\&A, 474, 145 